\title{
Quenching of vortex breakdown oscillations via harmonic modulation
}

\author{
J. M. LOPEZ $Z^{1}$, Y. D. CUI ${ }^{2}$, F. MARQUES AND T. T. LIM ${ }^{4}$ \\ ${ }^{1}$ Department of Mathematics and Statistics, Arizona State University, Tempe AZ 85287, USA \\ ${ }^{2}$ Temasek Laboratories, National University of Singapore, 119260 Singapore \\ ${ }^{3}$ Departament de Física Aplicada, Univ. Politècnica de Catalunya, Barcelona 08034, Spain \\ ${ }^{4}$ Department of Mechanical Engineering, National University of Singapore, 119260 Singapore
}

(Received 2 July 2007 and in revised form 12 December 2007)

Vortex breakdown is a phenomenon inherent to many practical problems, such as leading-edge vortices on aircraft, atmospheric tornadoes, and flame-holders in combustion devices. The breakdown of these vortices is associated with the stagnation of the axial velocity on the vortex axis and the development of a near-axis recirculation zone. For large enough Reynolds number, the breakdown can be timedependent. The unsteadiness can have serious consequences in some applications, such as tail-buffeting in aircraft flying at high angles of attack. There has been much interest in controlling the vortex breakdown phenomenon, but most efforts have focused on either shifting the threshold for the onset of steady breakdown or altering the spatial location of the recirculation zone. There has been much less attention paid to the problem of controlling unsteady vortex breakdown. Here we present results from a combined experimental and numerical investigation of vortex breakdown in an enclosed cylinder in which low-amplitude modulations of the rotating endwall that sets up the vortex are used as an open-loop control. As expected, for very low amplitudes of the modulation, variation of the modulation frequency reveals typical resonance tongues and frequency locking, so that the open-loop control allows us to drive the unsteady vortex breakdown to a prescribed periodicity within the resonance regions. For modulation amplitudes above a critical level that depends on the modulation frequency (but still very low), the result is a periodic state synchronous with the forcing frequency over an extensive range of forcing frequencies. Of particular interest is the spatial form of this forced periodic state: for modulation frequencies less than about twice the natural frequency of the unsteady breakdown, the oscillations of the near-axis recirculation zone are amplified, whereas for modulation frequencies larger than about twice the natural frequency the oscillations of the recirculation zone are quenched, and the near-axis flow is driven to the steady axisymmetric state. Movies are available with the online version of the paper.

\section{Introduction}

Swirling vortex flows such as are found on swept-wing aircraft at high angles of attack, in turbomachinary and swirl combusters, and in tornadoes are susceptible to vortex breakdown, a sudden and drastic bursting of the vortex often accompanied by localized regions of recirculation on the swirl axis. Vortex breakdown has been the subject of intense study for the past half-century, and although there has been significant progress in our understanding of the flow phenomenon, much remains unclear. Extensive reviews on this subject include Hall (1972), Leibovich (1978), 
Escudier (1988), Delery (1994), Lucca-Negro \& O'Doherty (2001). Depending on the practical application, the occurrence of vortex breakdown can have either favourable or detrimental effects, and there is much interest in controlling the phenomenon.

Controlling vortex breakdown has received significant attention for swept-wing aircraft at high angles of attack, where the lift is primarily generated by the vortices shed from the delta wings or leading-edge extensions. A characteristic of these vortices is that above a critical angle of attack, they suffer vortex breakdown, and the associated unsteadiness induces unsteady buffet loads on the aircraft's vertical stabilizers, leading to premature fatigue failure. There have been numerous studies on how to control and/or modify the breakdown of these vortices in order to alleviate the tail buffeting (see Mitchell \& Délery 2001, for a comprehensive review), but for the most part the strategies have focused on either shifting the location of the vortex breakdown or on inhibiting its occurrence. And yet, it seems that the problem is not that the vortex has broken down per se, but rather that the temporal characteristics of the unsteady vortex breakdown excite the tail buffeting. There have been very few investigations of control strategies on vortex breakdown where the goal has been to affect its temporal characteristics. Unsteady blowing on model aircraft has been explored, but such problems have so many variables due to the complex geometry that more generic fundamental studies are called for. A recent experimental investigation into the effects of periodic axial pulsing in a more idealized flow (Khalil, Hourigan \& Thompson 2006) has also focused on shifting the location of the breakdown.

Following the experimental study of Escudier (1984), the swirling vortical flow in the axis region of an enclosed cylinder driven by the rotation of an endwall has been a very popular setting for fundamental investigations of vortex breakdown. The confined geometry leads to a well-posed problem where steady axisymmetric vortex breakdown recirculation zones are readily realized in laboratory experiments and simulated numerically (e.g. Lopez 1990). More recently, this flow geometry has been used to investigate a number of strategies for the control of the steady axisymmetric vortex breakdown. Herrada \& Shtern (2003) numerically investigated the thermal suppression of steady axisymmetric vortex breakdown by means of an imposed axial temperature gradient which induces via centrifugal convection a large-scale meridional circulation opposing (or enhancing, depending on the sign of the temperature gradient) the recirculation on the axis. Earlier, using the Boussinesq approximation, Lugt \& Abboud (1987) also showed that an imposed axial temperature gradient could suppress steady axisymmetric vortex breakdown. Husain, Shtern \& Hussain (2003) experimentally studied the control of steady axisymmetric vortex breakdown by the co- or counter-rotation of a slender rod placed along the axis. Mununga et al. (2004) showed experimentally that a small differentially rotating disk embedded into the stationary endwall could be used to effect a similar control of the steady axisymmetric vortex breakdown. All of these open-loop control studies were restricted (by design) to parameter regimes where, in the absence of the control strategies, the flow was steady and axisymmetric. Gallaire, Chomaz \& Huerre (2004) conducted a closed-loop control study of vortex breakdown in an idealized pipe flow with the objective being to stabilize the steady axisymmetric columnar vortex, i.e. to suppress the onset of vortex breakdown.

In this study, we investigate the open-loop control of unsteady vortex breakdown in the confined cylinder geometry. The control mechanism is provided by a forced harmonic modulation of the rate of rotation of the rotating endwall. The investigation is both numerical and experimental. For the unforced flow, it is well known that for cylinders of height-to-radius aspect ratio between about 1.6 and 2.8, the onset of 
unsteadiness as the rate of rotation of the endwall (measured non-dimensionally by the Reynolds number) increases is via a supercritical axisymmetric Hopf bifurcation (Gelfgat, Bar-Yoseph \& Solan 2001), and that the resultant time-periodic axisymmetric flow is stable to three-dimensional perturbations for a considerable range of Reynolds numbers beyond onset (Blackburn \& Lopez 2000, 2002; Blackburn 2002; Lopez, Cui \& Lim 2006; Lopez 2006). Here, we report in detail on the response to variations in the forcing amplitude and forcing frequency for a time-periodic axisymmetric state in a cylinder of aspect ratio 2.5 at a Reynolds number of 2800 , which is characterized by a large double vortex breakdown bubble undergoing largeamplitude pulsations along the axis. For very small forcing amplitudes, the resultant flow is quasi-periodic, possessing both the natural frequency of the unforced bubble and the forcing frequency. As the amplitude is increased to between $2 \%$ and $5 \%$ (depending irregularly on the forcing frequency), the resultant flow locks onto the forcing frequency and the natural frequency is completely suppressed. This is a common result in periodically forced flows (Chiffaudel \& Fauve 1987). But what is particularly interesting in this case is how the spatial nature of the forced limit cycle (locked to the forcing frequency) changes with the forcing frequency. For low forcing frequencies (less than about twice the natural frequency), the forced limit cycle consists of an enhanced vortex breakdown recirculation bubble on the axis oscillating with larger amplitude than in the unforced case, whereas for larger forcing frequencies, the locked limit cycle has a (nearly) stationary vortex breakdown bubble on the axis, and its oscillations are most pronounced near the cylinder sidewall. We have also found windows of limit cycles locked to half the forcing frequency. Both the experiments and the numerical simulations indicate that all these flow phenomena remain axisymmetric, at least for Reynolds numbers less than about 3000 .

\section{Governing equations and numerical scheme}

We consider the flow in a circular cylinder of radius $R$ and depth $H$, with the bottom lid rotating at a modulated rate $\Omega\left(1+A \sin \left(\Omega_{f} t^{*}\right)\right)$ where $t^{*}$ is dimensional time in seconds, $\Omega \mathrm{rad} \mathrm{s}^{-1}$ is the mean rotation, $\Omega_{f} \mathrm{rad} \mathrm{s}^{-1}$ is the forcing frequency, and $A$ is the relative forcing amplitude. The system is non-dimensionalized using $R$ as the length scale, and the dynamic time $1 / \Omega$ as the time scale. There are four non-dimensional parameters:

$$
\begin{array}{ll}
\text { Reynolds number: } & R e=\Omega R^{2} / \nu, \\
\text { forcing amplitude: } & A, \\
\text { forcing frequency: } & \omega_{f}=\Omega_{f} / \Omega, \\
\text { aspect ratio: } & H / R,
\end{array}
$$

where $v$ is the kinematic viscosity. The non-dimensional cylindrical domain is $(r, \theta$, $z) \in[0,1] \times[0,2 \pi) \times[1, H / R]$. The resulting non-dimensional governing equations are

$$
\left(\partial_{t}+\boldsymbol{u} \cdot \nabla\right) \boldsymbol{u}=-\nabla p+\frac{1}{R e} \nabla^{2} \boldsymbol{u}, \quad \nabla \cdot \boldsymbol{u}=0,
$$

where $\boldsymbol{u}=(u, v, w)$ is the velocity field and $p$ is the kinematic pressure.

The boundary conditions for $\boldsymbol{u}$ are:

$$
\begin{array}{ll}
r=1: & u=v=w=0, \\
z=H / R: & u=v=w=0, \\
z=0: & u=w=0, \quad v=r\left(1+A \sin \left(\omega_{f} t\right)\right) .
\end{array}
$$


Regularity conditions (i.e. that the velocity be analytic) on the axis $(r=0)$ are enforced using appropriate spectral expansions for $\boldsymbol{u}$, and the discontinuity in azimuthal velocity at the bottom corner has been regularized in order to achieve spectral convergence.

\subsection{Numerical method}

The governing equations have been solved using the second-order time-splitting method proposed in Hughes \& Randriamampianina (1998) combined with a pseudospectral method for the spatial discretization, utilizing a Galerkin-Fourier expansion in the azimuthal coordinate $\theta$ and Chebyshev collocation in $r$ and $z$. The radial dependence of the variables is approximated by a Chebyshev expansion in $[-1,+1]$ and enforcing their proper parities at the origin (Fornberg 1998). Specifically, the vertical velocity $w$ has even parity $w(-r, \theta, z)=w(r, \theta+\pi, z)$, whereas $u$ and $v$ have odd parity. To avoid including the origin in the collocation mesh, an odd number of Gauss-Lobatto points in $r$ is used and the equations are solved only in the interval $(0,1]$. Following Orszag \& Patera (1983), we have used the combinations $u_{+}=u+\mathrm{i} v$ and $u_{-}=u-\mathrm{i} v$ in order to decouple the linear diffusion terms in the momentum equations. For each Fourier mode, the resulting Helmholtz equations for $w, u_{+}$and $u_{-}$have been solved using a diagonalization technique in the two coordinates $r$ and $z$. The imposed parity of the functions guarantees the regularity conditions at the origin needed to solve the Helmholtz equations (Mercader, Net \& Falqués 1991).

In this study, we have fixed $H / R=2.5$ and consider variations in $R e, A$ and $\omega_{f}$. We have used 96 spectral modes in $z, 64$ in $r$, and up to 24 in $\theta$ for non-axisymmetric computations, and a time step $\mathrm{d} t=2 \times 10^{-2}$ dynamic time units.

\section{Experimental apparatus and technique}

The current experiments were performed using the same apparatus as in Lopez et al. (2006), but with the experimental procedures modified for this particular study. The apparatus is shown schematically in figure 1. It is inverted from the actual experimental setup for ease of comparison with the experimental results of others which have the rotating disk at the bottom of the cylinder (flow visualization photos are also inverted). A detailed description of the apparatus can be found in Lopez et al. (2006), and only the essential features are described here.

The apparatus consists of a Plexiglas cylinder, with a matching rotating disk at the bottom and a stationary disk at the top of the cylinder. The cylinder was fabricated from a solid piece of Plexiglas rod and painstakingly polished to optical quality. The inner radius is $R=8.625 \pm 0.005 \mathrm{~cm}$ and the wall thickness is $2.1 \mathrm{~cm}$. The rotating disk sits neatly on a high-precision thrust bearing mounted on an adjacent fixed plate, which in turn is push-fitted into the bottom end of the cylinder to ensure accurate alignment. The edge of the rotating disk has a maximum excursion of $0.040 \mathrm{~mm}$ (about $0.03^{\circ}$ ) and a nominal gap of $0.40 \mathrm{~mm}$ between the rotating plate and the cylinder. The disk was driven by a micro-stepper motor operating at 20000 steps per rev, with an adjustable speed range of up to 240 r.p.m. $\left(\Omega=25.1 \mathrm{rad} \mathrm{s}^{-1}\right)$. The motor was controlled by software written in Labview, which allows the bottom disk to rotate at a modulated rate $\Omega\left(1+A \sin \left(\Omega_{f} t^{*}\right)\right)$. Most of the experiments are at $R e=2800$ with $A$ varying from 0.002 to 0.09 . Although the height $H$ of the flow domain can be varied infinitesimally by changing the position of the stationary top disk using a $1.0 \mathrm{~mm}$ pitched screw stud, the aspect ratio was maintained at a constant $H / R=2.5$.

The working fluid was a mixture of glycerin and water (roughly $74 \%$ glycerin by weight) with kinematic viscosity $v=0.254 \pm 0.002 \mathrm{~cm}^{2} \mathrm{~s}^{-1}$ at a room temperature of 


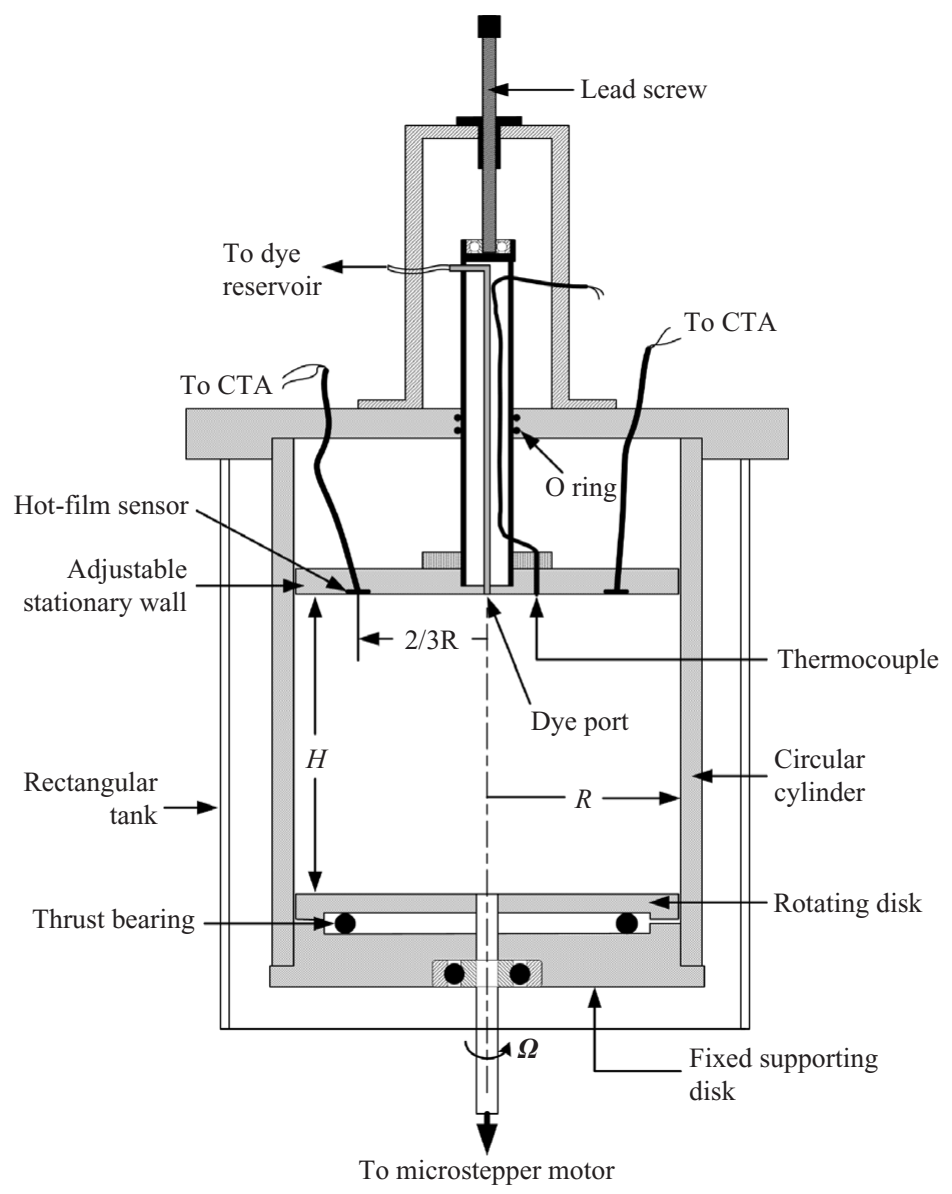

FIGURE 1. Schematic of the flow apparatus.

$22.3^{\circ} \mathrm{C}$. In all cases, the viscosity was measured using a Hakke Rheometer to an accuracy of about $0.8 \%$, and the temperature of the mixture was monitored regularly using a thermocouple located at the bottom of the cylinder to the accuracy of $0.05^{\circ} \mathrm{C}$, giving a maximum uncertainty in the Reynolds number of about \pm 22 in absolute value. To minimize flow image distortion due to the curvature of the cylinder, the whole cylinder was immersed in a rectangular Plexiglas box filled with the same working fluid (both the solution and the Plexiglas have similar refractive indices).

Because the cylinder wall is too thick to allow efficient heat exchange between the fluid inside the cylinder and its surroundings, there is a gradual increase in the temperature of the fluid, resulting in an increase in $R e$ at a rate $\partial R e / \partial t \approx 25$ per hour. In the present investigation, this is not significant as each data point required about 20 minutes of running time which translates into less than $0.3 \%$ change in $R e$. However, after each data point was taken, the experiment was halted to allow the fluid to cool down to the room temperature of $22.3^{\circ} \mathrm{C}$ before commencing the next experiment.

To measure the oscillatory behaviour of the flow, two flush-mounting hot films (Dantec 55 R47) were attached to the surface of the stationary endplate with waterproof glue. The nominal thickness of the sensor is less $0.1 \mathrm{~mm}$, and therefore its effect on the flow was negligible. These two sensors were located at $2 / 3$ of the radius of the cylinder and $180^{\circ}$ apart. As in Lopez et al. (2006), the hot films were not calibrated, 
(a)

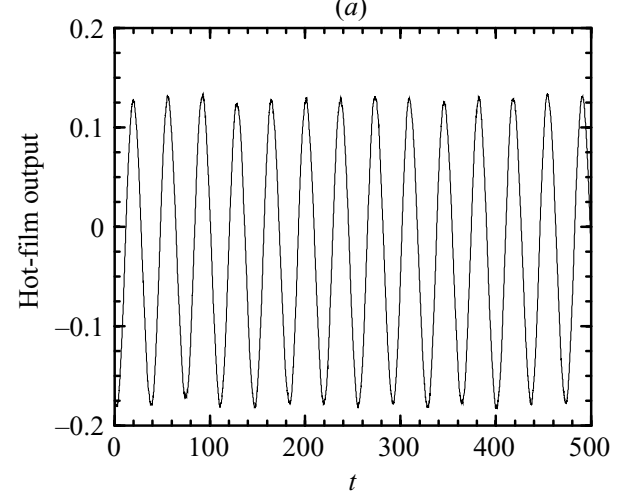

(b)

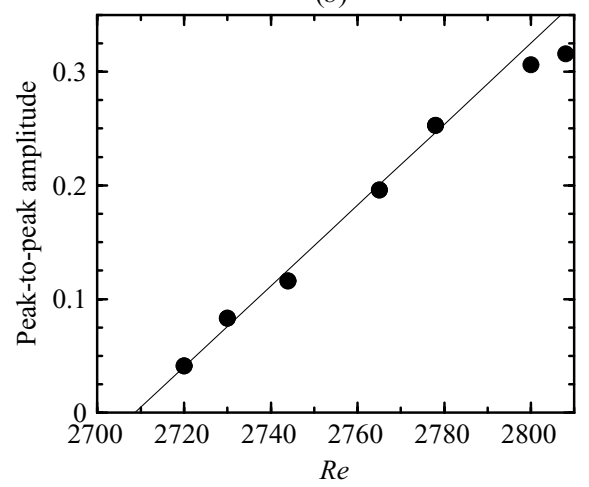

FIGURE 2. (a) Time series of hot-film output at $H / R=2.5$ and $R e=2800$, and $(b)$ variation with $R e$ of the peak-to-peak amplitude of the hot-film output, both for the natural (unmodulated) limit cycle state $L C_{N}$.

primarily due to the design of the glue-on hot film which makes calibration against a known flow velocity difficult; once the hot film is glued to a surface, it cannot be easily removed (without damage) for calibration in another facility. Nevertheless, calibration is not of concern when measuring the temporal frequencies in a flow, and this is confirmed by the experimental results reported in Lopez et al. (2006). Given that the frequencies of interest in the present investigation are below $1 \mathrm{~Hz}$, the output signal of the hot film from the constant-temperature anemometer (CTA; Dantec $55 \mathrm{M} 01$ ) was conditioned by a low-pass filter with a cutoff frequency of $10 \mathrm{~Hz}$ to eliminate high-frequency noise before it was amplified with an analog amplifier. The output signal was sampled at $100 \mathrm{~Hz}$ using a computer for subsequent analysis. Although laser Doppler anemometry (LDA) was not attempted in the present study owing to the equipment not being available in our laboratory, past studies have shown that hot-film or hot-wire anemometry is as good as or even better than LDA when measuring oscillatory behaviour for a long time.

\section{Results}

\subsection{The natural limit cycle $L C_{N}$}

The objective of this study is to explore the effects of an imposed harmonic forcing on an oscillatory vortex breakdown state. We shall begin by briefly reviewing the salient characteristics of this state (which we shall refer to as the natural limit cycle $L C_{N}$ ) and establishing the fidelity of the experimental apparatus in obtaining it.

Escudier (1984) first reported the $L C_{N}$ state in his experiments, noting its axisymmetric nature over a wide range of aspect ratios and Reynolds numbers. Gelfgat et al. (2001) showed numerically that the onset of $L C_{N}$ is via a supercritical axisymmetric Hopf bifurcation for $H / R \in(1.6,2.8)$. Nonlinear computations (Blackburn \& Lopez 2000, 2002) have shown that this oscillatory state remains stable to three-dimensional perturbations for $R e$ up to about 3400. That numerical finding is consistent with the experimental observations of Stevens, Lopez \& Cantwell (1999). These studies (as well as others, such as Lopez, Marques \& Sanchez 2001) have estimated the critical $R e$ for the Hopf bifurcation at $H / R=2.5$ to be about 2710 , and the period of oscillation to be about 36 (using $\Omega$ as the time scale). Figure 2(a) shows hot-film output over several cycles of the natural limit cycle flow at $H / R=2.5$ 
and $R e=2800$. Using the peak-to-peak amplitude of the hot-film signal as a measure of the flow state, figure $2(b)$ shows its variation with $R e$; a simple extrapolation to zero gives the experimental estimate $R e_{c}=2710$ which is also in excellent agreement with the theoretical estimate.

Any physical experiment will have small imperfections and perturbations which are not axisymmetric, and the question is whether these imperfections affect the dynamics, i.e. do they render the resulting flow non-axisymmetric? There has been much discussion on this matter in the literature (e.g. Sotiropoulos \& Ventikos 2001; Sotiropoulos, Webster \& Lackey 2002; Ventikos 2002; Thompson \& Hourigan 2003; Brons et al. 2007) where the studies have imposed imperfections in order to account for the asymmetric dye-streak visualizations in experiments. In a time-periodic axisymmetric flow, free of any imperfections, if the dye (or any passive scalar) is not released axisymmetrically, the resulting dye-sheet will not be axisymmetric (Lopez \& Perry 1992b; Hourigan, Graham \& Thompson 1995). Flow visualization is not appropriate for determining whether such a flow is axisymmetric or not. The important point is that if axisymmetry ( $S O(2)$ symmetry to be precise) is broken, the non-axisymmetric pattern will precess at the Hopf frequency responsible for the symmetry-breaking (Iooss \& Adelmeyer 1998; Crawford \& Knobloch 1991; Knobloch 1996). This means, for example, that the hot-film time-series from our experiment should pick up a signal corresponding to such a precession if the flow were not axisymmetric. No such signal was detected. The spectra of hundreds of experiments at various points in parameter space (only a select few are shown in the paper) only show signals at the natural frequency and the modulation frequency and their linear combinations. This, together with the results shown in figure 2 for the unmodulated cases, indicate that any small imperfections in our experiment do not result in nonaxisymmetric flow. However, owing to unavoidable imperfections in the release of dye, the visualized dye sheets shown are slightly asymmetric (e.g. see figures 3 and 17 with small deviations and figure 15 with larger deviations from axisymmetry).

\subsection{Harmonic forcing of $L C_{N}$ : temporal characteristics}

The issue being addressed in this paper is the response of a time-periodic vortex breakdown flow, $L C_{N}$, to harmonic forcing. $L C_{N}$ exists and is stable over a wide range of $(R e, H / R)$ parameter space; the frequency of its oscillation (non-dimensionalized by $\Omega$ ) is essentially independent of $R e$ and only varies slightly with $H / R$ (Stevens et al. 1999; Lopez et al. 2001; Gelfgat et al. 2001; Blackburn \& Lopez 2002). In this study, we present detailed results over a wide sweep of the two control parameters, $A$ and $\omega_{f}$ the amplitude and frequency of the harmonic forcing, on $L C_{N}$ at $R e=2800$ and $H / R=2.5$. This state is a little beyond critical, with $\epsilon=\left(R e-R e_{c}\right) / R e_{c} \approx 0.0332$. The results are qualitatively similar at other $(R e, H / R)$ values where $L C_{N}$ is the primary bifurcating mode from the basic state, and the results presented are not peculiar to the choice $R e=2800$ and $H / R=2.5$.

Flow visualization (using food dye) of $L C_{N}$ over one period is shown in figure 3 (available with the online version of the paper is a movie showing $L C_{N}$ over about four oscillation periods, movie 1). The pulsing of the recirculation zone on the axis and the formation and folding of lobes every period are clearly evident and follow the detailed description of the chaotic advection given in Lopez \& Perry (1992a) for this flow. Using hot film measurements at $R e=2800$, we find the natural frequency of the oscillator (scaled by the rotation frequency of the disk $\Omega$ ) to be $\omega_{0}=0.1735$ (giving a period of 36.2), which is in good agreement with previous estimates of the Hopf frequency and with the numerically determined natural frequency of $L C_{N}$ in 


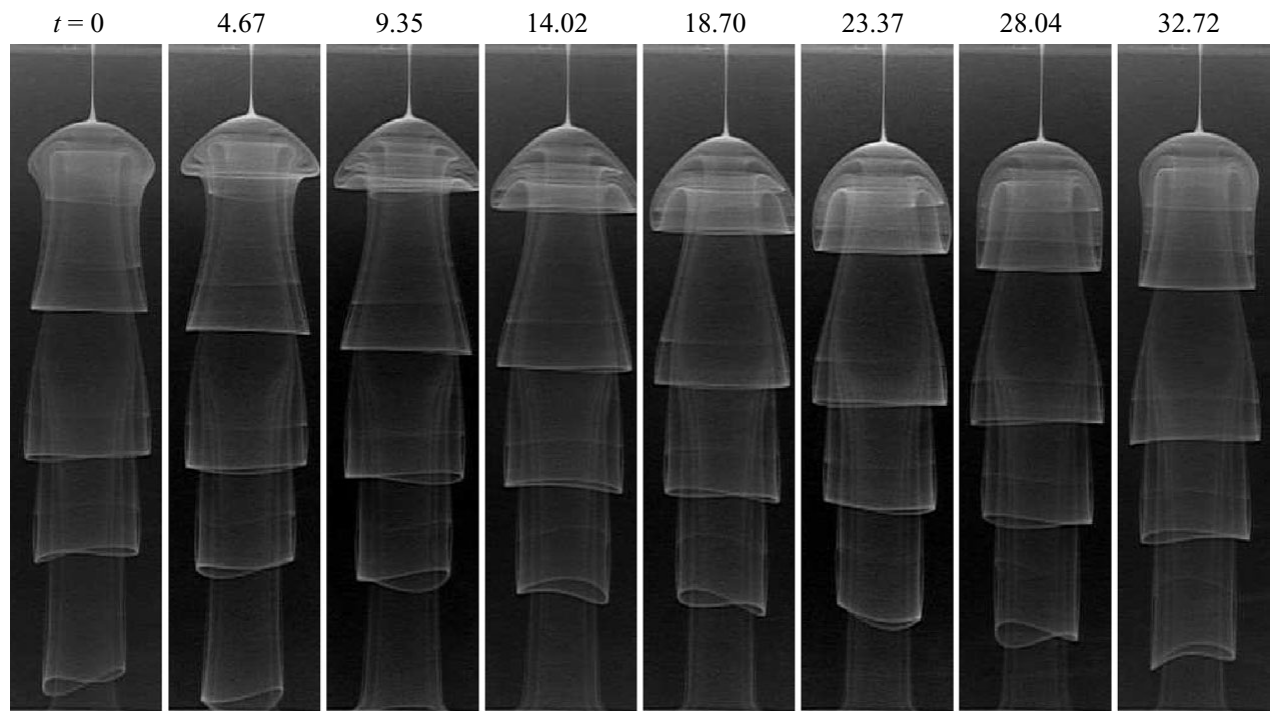

Figure 3. Dye flow visualization of the central core region of $L C_{N}$ at $H / R=2.5$ and $R e=2800$ at various times; the period is about 36.2 (the time for the first frame has been arbitrarily set to zero).

this study. The natural frequency of $L C_{N}, \omega_{n}$, is a (weak) function of the parameters of the problem, including the amplitude and frequency of the modulation; we will use $\omega_{0}=\omega_{n}(R e=2800, H / R=2.5, A=0)$ for scaling purposes.

Periodically forced limit cycles are often studied by varying the forcing amplitude $A$ and the forcing frequency $\omega_{f}$. Figure 4 shows experimental time series and their corresponding power spectral density, as the forcing amplitude increases from zero with a forcing frequency not in resonance with the natural frequency (in this case, $\omega_{f}=0.1$, so $\left.\omega_{f} / \omega_{0} \approx 0.576\right)$. The experimental time series are from hot film output data. Figure 4(a) is simply $L C_{N}$ at $A=0$, a periodic solution with a single frequency $\omega_{n}=\omega_{0}$ and its harmonics in the power spectral density. For $A<0.03$, the flow is quasi-periodic, $Q P$, with two frequencies $\omega_{f}$ and $\omega_{n}$. As $A$ increases, the relative strength of the spectral energies of the two frequencies shifts from $\omega_{n}$ to $\omega_{f}$, and by $A=0.030$, the power in the spectra at $\omega=\omega_{n}$ goes to zero and the flow is a limit cycle synchronous with the forcing, $L C_{F}$. When $\omega_{f} / \omega_{n}$ is not too close to a rational value $p / q$ with $q \leqslant 4$, this scenario is typical of what is observed.

Figure 5 shows phase portraits of the numerical solutions as the forcing amplitude increases from zero, for the same values of the remaining parameters as in figure 4: $H / R=2.5, R e=2800$ and $\omega_{f}=0.1$. It illustrates the same sequence of events: the natural limit cycle $L C_{N}$ for $A=0$ bifurcates to a quasi-periodic solution $Q P$ densely filling a two-torus $\mathbb{T}^{2}$ when $A$ is increased from 0 , and at about $A \approx 0.0290$ this $Q P$ solution bifurcates to the forced limit cycle $L C_{F}$. Phase portraits of the numerical solutions are drawn in terms of the vertical velocity at two different points: $W_{a}=w(r=0.20, z=0.75 H / R)$ close to the vortex breakdown bubble and $W_{w}=w(r=0.70, z=0.75 H / R)$ at the jet emerging from the sidewall-rotating disk corner.

The bifurcation from a limit cycle to a quasi-periodic solution (evolving on an invariant two-torus $\mathbb{T}^{2}$ ) is called a Neimark-Sacker bifurcation; it is a Hopf bifurcation of limit cycles, described for example in Kuznetsov (2004). The bifurcation 
(a) $A=0$
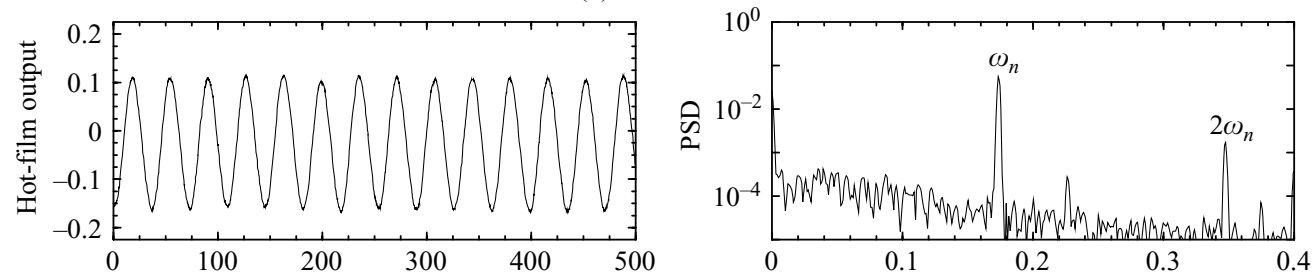

(b) $A=0.01$
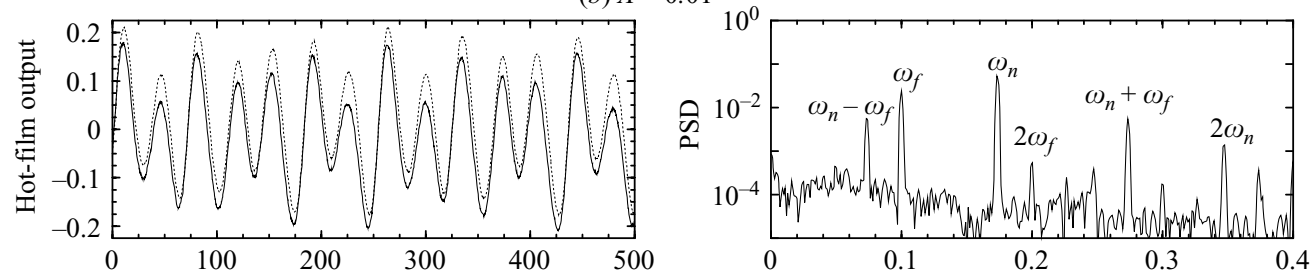

(c) $A=0.02$
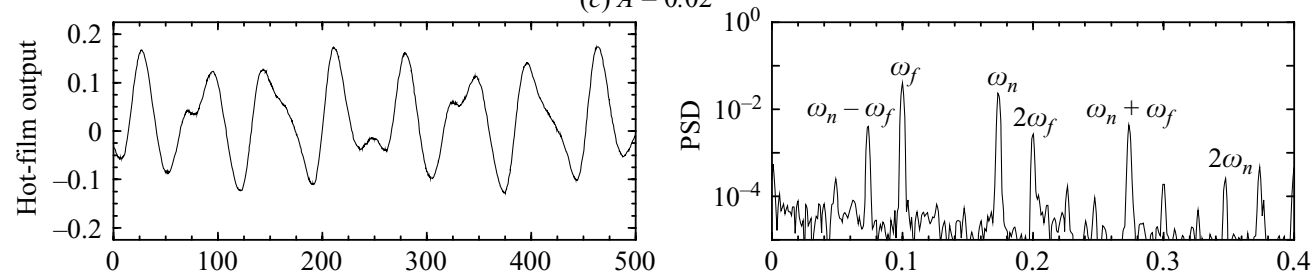

(d) $A=0.03$
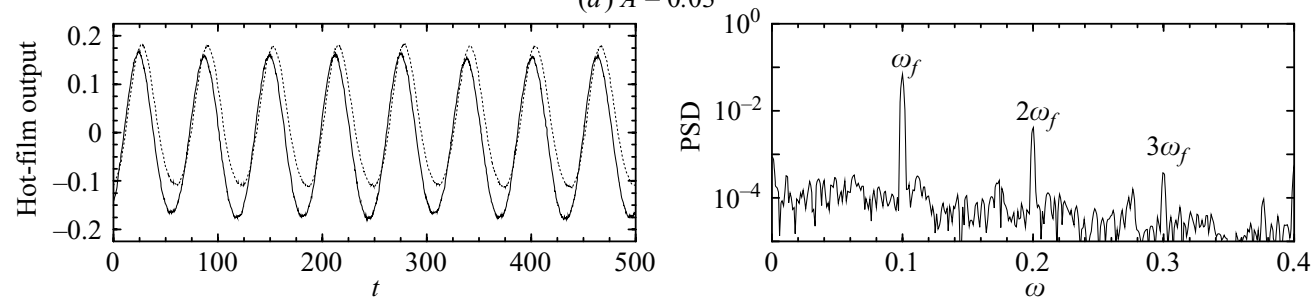

FIGURE 4. Hot film output time series and corresponding power spectral density for $H / R=2.5$, $R e=2800$ with forcing frequency $\omega_{f}=0.1$ and forcing amplitude $A$ as indicated. In $(b)$ and $(d)$ the hot film outputs from both channels are plotted.
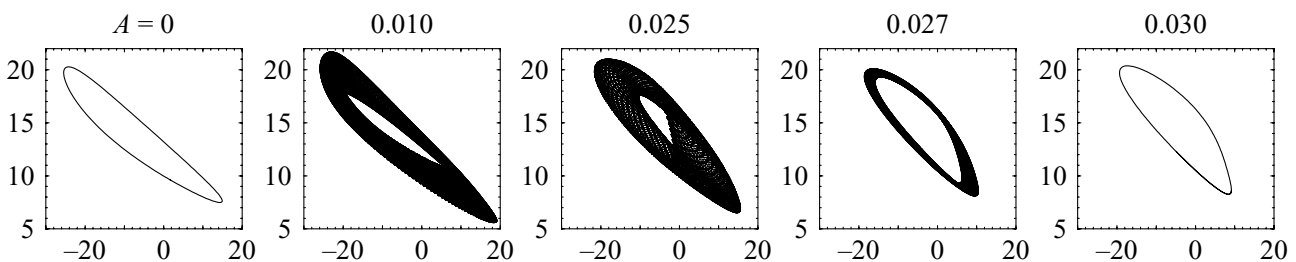

FIGURE 5. Phase portraits (with $W_{a}$ and $W_{w}$ as the horizontal and vertical axes) of the numerical solutions at $R e=2800, H / R=2.5, \omega_{f}=0.10\left(\omega_{f} / \omega_{0} \approx 0.576\right)$ and $A$ as indicated.

to a $\mathbb{T}^{2}$ is a codimension-one phenomenon: it takes place with the variation of a single parameter of the dynamical system (e.g. the amplitude $A$ in the bifurcations shown in figures 4 and 5). However, the dynamics on the two-torus needs a second parameter to be described in detail, and the forcing frequency $\omega_{f}$ is used as the second parameter; 
(a)

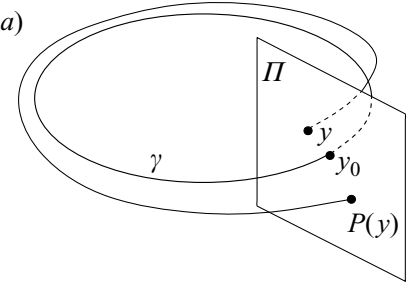

(b)

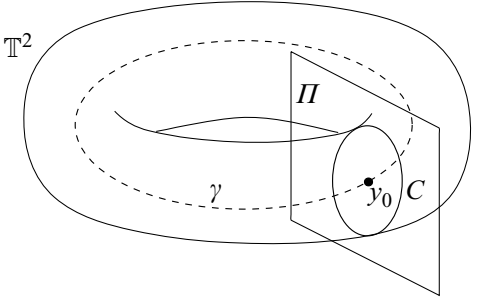

FiguRE 6. (a) Schematic of the Poincare map, showing the stable limit cycle $\gamma$ and a trajectory starting at a point $y$ in $\Pi$ near the limit cycle $y_{0}$. (b) Poincaré map after a Neimark-Sacker bifurcation of $\gamma$, spawning a two-torus $\mathbb{T}^{2}$, whose cross-section in $\Pi$ is the invariant circle $C$.

in the $\left(A, \omega_{f}\right)$-parameter space, the Neimark-Sacker bifurcation takes place along a curve. The dynamics on $\mathbb{T}^{2}$ can be reduced to the study of families of circle maps (Arnold 1983). One of the salient features of the Neimark-Sacker bifurcation is the presence of Arnold tongues (resonance horns); these are regions in $\left(A, \omega_{f}\right)$-parameter space emanating from points on the Neimark-Sacker bifurcation curve at which the two frequencies $\omega_{f}$ and $\omega_{n}$ are in rational ratios. Each horn is characterized by a phase-locked solution for which the winding number $\omega_{f} / \omega_{n}=p / q$, for some integers $p$ and $q$. In between the horns, emerging from all irrational points on the Neimark-Sacker bifurcation curve, there are curves corresponding to quasi-periodic solutions with frequencies $\omega_{f}$ and $\omega_{n}$ in irrational ratios. For a detailed description of the Neimark-Sacker bifurcation see, for example, Arrowsmith \& Place (1990). The dynamics in small neighborhoods of the resonances along the Neimark-Sacker curve can be very complicated, in particular when one of the integers $p$ or $q$ is small (strong resonances, see Kuznetsov 2004). There have been significant advances in the numerical investigation of the dynamics in these neighborhoods (e.g. Schilder \& Peckham 2007), but for the most part only low-dimensional ODE model problems have been tackled.

In our problem, on increasing $A$ from zero, there are two different NeimarkSacker bifurcations. The corresponding curves in $\left(A, \omega_{f}\right)$-parameter space have been numerically and experimentally determined, and are illustrated for $H / R=2.5$, $R e=2800$ in figure 7 below. The solid curve in this figure corresponds to the NeimarkSacker bifurcation from the $Q P$ state collapsing onto the $L C_{F}$, which is the observed solution for large forcing amplitude $A$. The other Neimark-Sacker bifurcation curve is the $A=0$ axis, where the natural limit cycle $L C_{N}$ bifurcates to a two-torus $Q P$ as $A$ increases from zero. This Neimark-Sacker bifurcation is slightly different from the standard one as described, for example, by Kuznetsov (2004), because having $A=0$ simplifies some of the dynamics; a detailed description of this case can be found in Gambaudo (1985).

The analysis of the dynamics in a neighbourhood of a periodic orbit $\gamma$, of period $T$, in a continuous system is greatly simplified by the introduction of the Poincare map. Let $\Pi$ be a hyperplane transverse to the orbit and $y_{0}$ be the point of intersection of $\Pi$ and $\gamma$ (see figure $6 a$ ). By continuity, the points on $\Pi$ in a neighbourhood $U_{0}$ of $y_{0}$ return to $\Pi$ after a time close to $T$. This defines a Poincaré map $P: U_{0} \rightarrow \Pi$. This Poincare map is equivalent to strobing the solutions with the frequency of the periodic orbit $\gamma$. Generically, the Poincare map is defined locally, in a neighbourhood of the periodic orbit considered. The periodic orbit $\gamma$ becomes a fixed point of $P$, and for a Neimark-Sacker bifurcation, the emerging two-torus $\mathbb{T}^{2}$ becomes an invariant circle $C$, and the $Q P$ solutions become circle maps (a schematic is shown in figure $6 b$ ). 

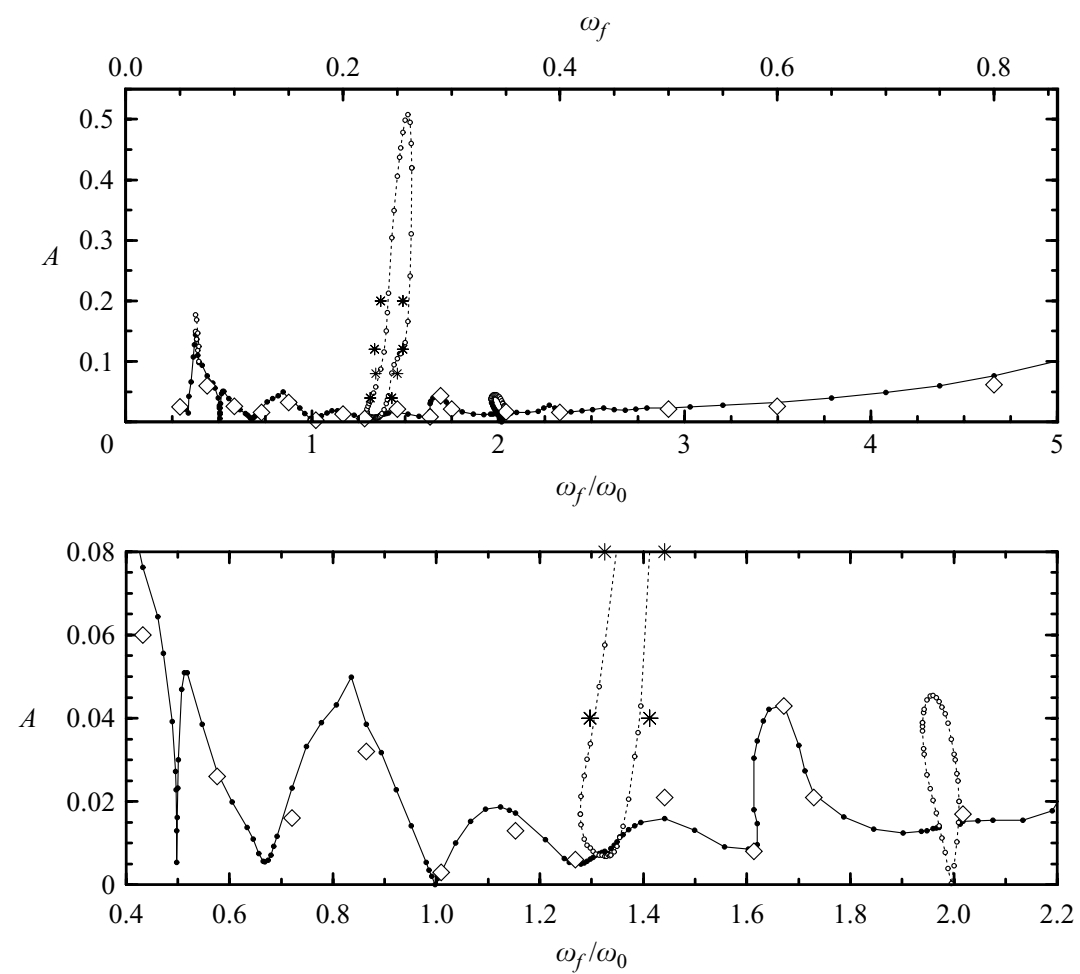

FiguRE 7. Critical forcing amplitude, $A_{\mathrm{c}}$, versus the forcing frequency $\omega_{f}$, and versus $\omega_{f} / \omega_{0}$, at $R e=2800$ and $H / R=2.5 ;(b)$ is an enlargement of $(a)$ highlighting some of the resonance horns. The small solid symbols are the numerically determined loci of Neimark-Sacker bifurcations (the curve joining these symbols is only to guide the eye), and the open diamonds are the corresponding experimental estimates. Below the Neimark-Sacker curve the $Q P$ state is observed, above it $L C_{F}$ is observed. In the regions enclosed by the dotted curves and open circles (there are three, near $\omega_{f} / \omega_{0} \approx 1 / 3,4 / 3$, and $2 / 1$ ) the flow is locked to a limit cycle with frequency $0.5 \omega_{f}$, and the star symbols are experimentally determined edges of the period-doubled region near $\omega_{f} / \omega_{0}=1.33$.

For the Neimark-Sacker bifurcation $L C_{N} \rightarrow Q P$ on the $A=0$ axis, the associated Poincare map $P_{0}$ is based on the natural frequency $\omega_{n}$ of $L C_{N}$. This map is local in nature, defined only in a neighbourhood of $L C_{N}$. Moreover, the frequency $\omega_{n}$ of $Q P$, which is inherited from $L C_{N}$ at the bifurcation, is a function of $A, \omega_{f}$ and the remaining parameters of the problem $(R e$ and $H / R)$; in particular, at the second Neimark-Sacker bifurcation $Q P \rightarrow L C_{F}$ with non-zero $A$, the power in the spectra at $\omega_{n}$ vanishes to zero and the Poincare map associated with $\omega_{n}$ ceases to exist. For this second Neimark-Sacker bifurcation, the Poincare map associated with $\omega_{f}$ is global in nature, and is defined as strobing at the forcing frequency $\omega_{f}$; it is well-defined for any solution and any value of the parameters, and is currently used in periodically forced systems. We make extensive use of this stroboscopic map $P$ in this study.

Figure 7 shows a parametric portrait of the system over an extensive range of $\omega_{f} / \omega_{0}$ in $\left(A, \omega_{f} / \omega_{0}\right)$ parameter space. The small filled circles are the numerically determined Neimark-Sacker bifurcations from $L C_{F}$ to $Q P$; the open diamonds are the experimental estimates of the loci of this bifurcation. In the enlargement shown in figure $7(b)$, some of the principal resonance horns are clearly evident, particularly 
(a) $\omega_{f} / \omega_{0} \approx 1 / 3$

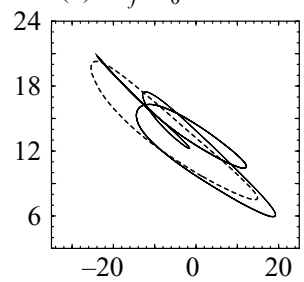

(b) $\omega_{f} / \omega_{0} \approx 1 / 2$

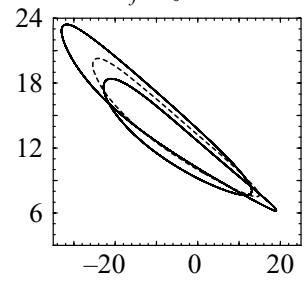

(c) $\omega_{f} / \omega_{0} \approx 1 / 1$

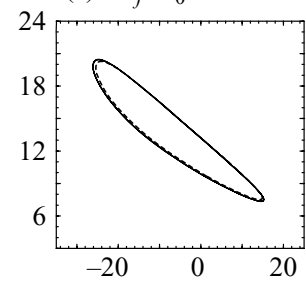

(d) $\omega_{f} / \omega_{0} \approx 2 / 1$

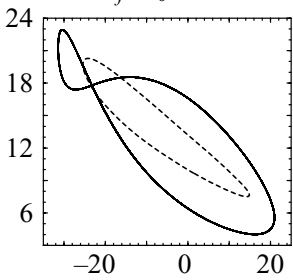

FIGURE 8. Phase portraits (with $W_{a}$ and $W_{w}$ as the horizontal and vertical axes) for $R e=2800, H / R=2.5, A=0.02$ and $\omega_{f} / \omega_{0}$ as indicated.

the $\omega_{f} / \omega_{0}=1 / 3,1 / 2,1 / 1$ and $2 / 1$ horns. Typical phase portraits of the numerically determined locked states inside these horns are shown in figure 8 ; the phase portrait of $L C_{N}$ is included in each as a dotted circuit for comparison. In the 1:3 horn, the phase portrait is of a limit cycle that undergoes three loops before closing in on itself; the time for it to close is about three times the period of $L C_{N}$. In the 1:2 horn, the locked state $L C_{L}$ executes two loops before closing, taking about two periods of $L C_{N}$ to do so. The locked state in the 1:1 horn is very little changed from $L C_{N}$. The stroboscopic map of $L C_{L}$ in these horns $(1: m)$ consists of a single fixed point (in contrast, using the local Poincare map based on $\omega_{n}$, their Poincare sections consist of $m$ period- $m$ points). In the 2:1 horn, the locked state $L C_{L}$ closes in on itself in one period of $L C_{N}$, but its stroboscopic map is not a single point, instead consisting of a pair of period-2 points; the locked state in this horn is not synchronous with $\omega_{f}$, instead it has period $4 \pi / \omega_{f}=2 \pi / \omega_{n}$.

Another feature in figure 7 is the presence of period-doubling bifurcation curves, shown as dotted curves. The small regions of period doubling close to the resonance horns $1: 3$ and $2: 1$ are associated with these horns, as we will show in detail later for the $2: 1$ case. However, the large period-doubling region near $\omega_{f} / \omega_{0} \approx 4 / 3$ is not directly related to the $4: 3$ horn. There is a very small overlap region between the period-doubling curve and the Neimark-Sacker bifurcation from $L C_{F}$ to $Q P$; the dynamics in this narrow region is very complicated and we have not explored it in detail, as we are focusing in this paper on controlling the vortex breakdown bubble oscillations. Figure 9 shows the period-doubling bifurcation as observed in the experiment from the hot-film output time series and their corresponding power spectral density. For $H / R=2.5, R e=2800$ and forcing amplitude $A=0.08$, the forcing frequency is increased from $\omega_{f}=0.22$ to 0.25 in steps of 0.01 , crossing the perioddoubling region. The additional peak at $\omega_{f} / 2$ is apparent in figures $9(b)$ and $9(c)$. Apart from noise, an additional low frequency $\omega_{*}$ is also observed, with an energy at least one order of magnitude smaller than the dominant peaks $\omega_{f}$ and $\omega_{f} / 2$. The origin of this peak is uncertain but we suspect it is associated with the fact that the modulation amplitude is large, and the inertia of the disk may be interfering with the harmonic forcing from the motor drive. In the numerics, no such low-frequency signal is observed, and the stroboscopic map is either a single fixed point outside the period-doubling region or a pair of period-2 points inside.

The large extent in $\left(A, \omega_{f}\right)$-space of the period-doubling region near $\omega_{f} / \omega_{0}=4 / 3$ suggests that it is not described by the harmonic forcing of an isolated limit cycle. We know from the linear stability analysis of the steady axisymmetric basic state (Lopez et al. 2001) that at $R e=2800$, a second limit cycle, $L C_{S}$, is about to bifurcate from the basic state (at about $R e=2850$ ), whose Hopf frequency $\omega_{s} \approx 0.67 \omega_{0}$. Forcing at 
(a) $\omega_{f}=0.22$

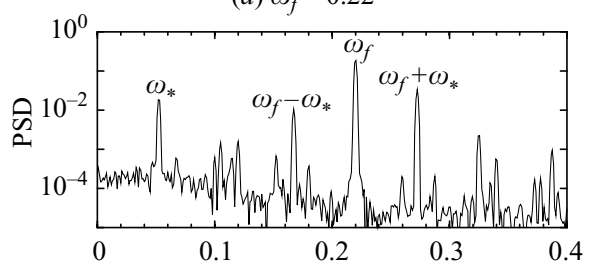

(c) $\omega_{f}=0.24$

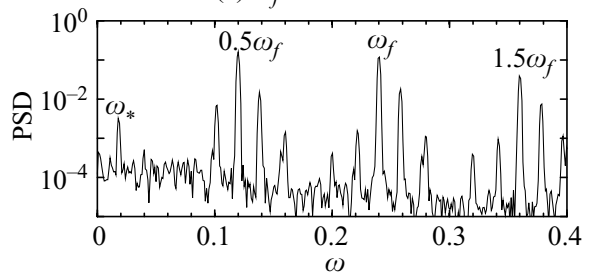

(b) $\omega_{f}=0.23$

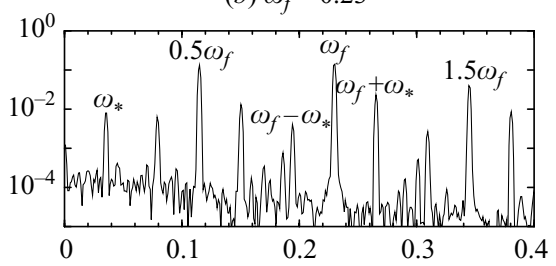

(d) $\omega_{f}=0.25$

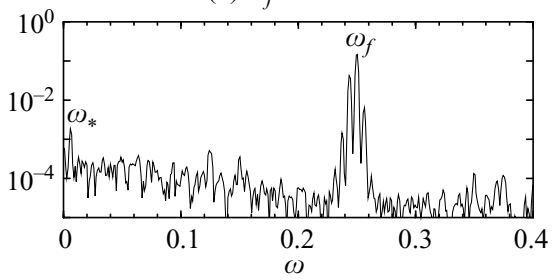

FIGURE 9. Hot film output time series and corresponding power spectral density for $H / R=2.5, R e=2800$ with forcing amplitude $A=0.08$ and forcing frequency $\omega_{f}$ as indicated.

$\omega_{f} \approx 1.33 \omega_{0}$ not only forces $L C_{N}$ at its $4: 3$ resonance, but $L C_{S}$ is also being forced at its $2: 1$ resonance. We conjecture that the large period-doubling region represents a nonlinear interaction between the 4:3 resonance of $L C_{N}$ and the 2:1 resonance of $L C_{S}$.

One of the first experimental studies in fluids where an oscillatory flow is harmonically forced to a periodic state synchronous with the forcing is that of Chiffaudel \& Fauve (1987). Their experiment consisted of a layer of mercury heated from below. Above a critical temperature difference across the layer, a Hopf bifurcation occurs to oscillatory convection rolls. This is their natural limit cycle $L C_{N}$. This is then harmonically forced by rotating the layer with a periodic angular velocity about the vertical axis. They considered $L C_{N}$ a little above critical, $\epsilon=0.023$, and generally for forcing amplitudes of only a few degrees the system become synchronous with the forcing, $L C_{F}$, except near strong resonance points. They examined in detail the 2:1 resonance horn region, both experimentally, and also theoretically. They derived an amplitude equation (essentially a continuous-time approximation to the normal form for the discrete-time map), and showed that in the neighbourhood of the 2:1 resonance only three kinds of states exist: the quasiperiod state $Q P$, the forced limit cycle $L C_{F}$, and the locked state, $L C_{L}$. The structure of their 2:1 resonance horn (their figure 3 ) is very similar to that of ours, shown in figure 10. Figure 10 consists of three bifurcation curves: the solid curves with filled circles are the Neimark-Sacker bifurcation curves separating $Q P$ and $L C_{F}$, the dashed curve with filled triangles is the period-doubling bifurcation curve separating $L C_{F}$ and $L C_{L}$, and the solid curves with filled squares are saddle-node-on-invariancecircle (SNIC) bifurcation curves on which the $Q P$ state synchronizes with the $L C_{L}$ state (for additional details on the SNIC bifurcations that define the borders of the Arnold tongues, see Arrowsmith \& Place 1990). The other symbols in the figure are loci of experimentally observed $Q P$ (open circles), $L C_{L}$ (filled diamonds) and $L C_{F}$ (open squares); their observed loci agree well with the delineations provided by the numerically determined bifurcation curves. Transients near the bifurcation curves are extremely slow, requiring thousands of forcing periods to determine the state numerically. Such slow transients are problematic experimentally as the Reynolds number drifts as the temperature slowly rises in the apparatus. 


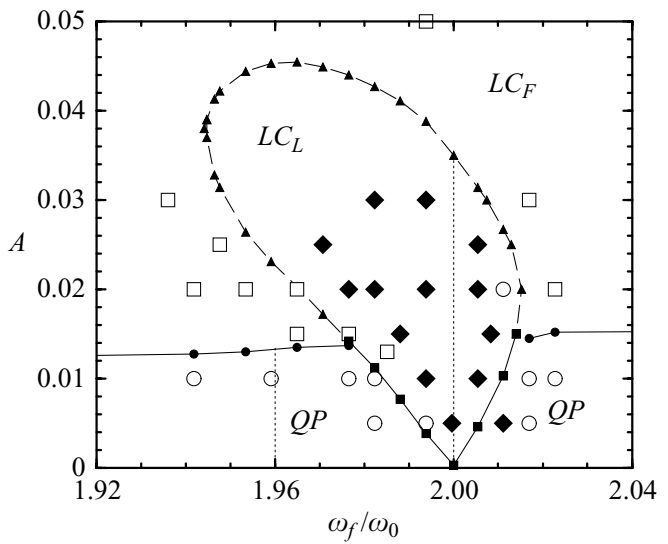

FiguRE 10. Enlargement of figure 7 near the 2:1 resonance horn. There are three bifurcation curves separating regions where the locked $L C_{L}$, the forced $L C_{F}$, and the quasi-periodic state $Q P$ are found. The solid curves with filled circles are the Neimark-Sacker bifurcation curves separating $Q P$ and $L C_{F}$, the dashed curve with filled triangles is the period-doubling bifurcation curve separating $L C_{F}$ and $L C_{L}$, and the solid curves with filled squares are saddle-node-on-invariance circle (SNIC) bifurcation curves on which the $Q P$ state synchronizes to the $L C_{L}$ state. The other symbols are loci of experimentally observed $Q P$ (open circles), $L C_{L}$ (filled diamonds) and $L C_{F}$ (open squares). The two dotted curves at $\omega_{f} / \omega_{0}=1.96$ and 2.0 are one-parameter paths along which the variation with $A$ in the power at $\omega_{n}$ and $\omega_{f}$ are shown in figure 13.

(a)

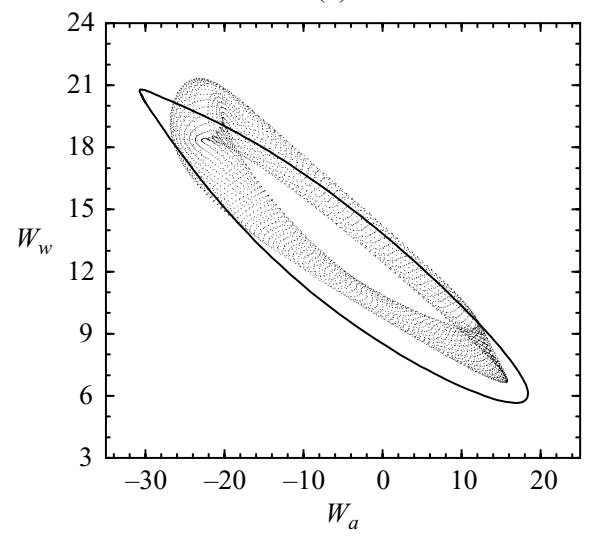

(b)

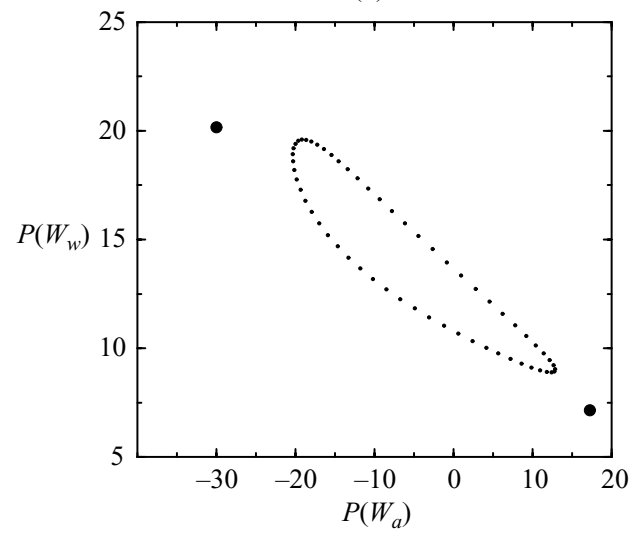

FIGURE 11. (a) Phase portraits in the neighbourhood of the $2: 1$ resonance for $Q P$ at $\omega_{f} / \omega_{0} \approx 1.965$ and $A=0.005$ just outside the resonance horn and for $L C_{L}$ at $\omega_{f} / \omega_{0}=2.0$ and $A=0.005$ inside the resonance horn; and $(b)$ are the corresponding Poincáre sections.

The phase portraits when crossing the Neimark-Sacker curve in the transition from $Q P$ to $L C_{F}$, are similar to the last two panels in figure 5. Figure 11(a) shows phase portraits at $A=0.005$ either side of the SNIC bifurcation; for $\omega_{f} \approx 1.965 \omega_{0}$ we see the two-torus structure of $Q P$ and for $\omega_{f}=2 \omega_{0}$ it has collapsed to the locked state $L C_{L}$. The SNIC nature of this transition is more clearly seen from the corresponding stroboscopic maps shown in figure 11(b). The stroboscopic map of the two-torus is an invariant circle exhibiting the characteristic slow-fast behaviour near the SNIC 


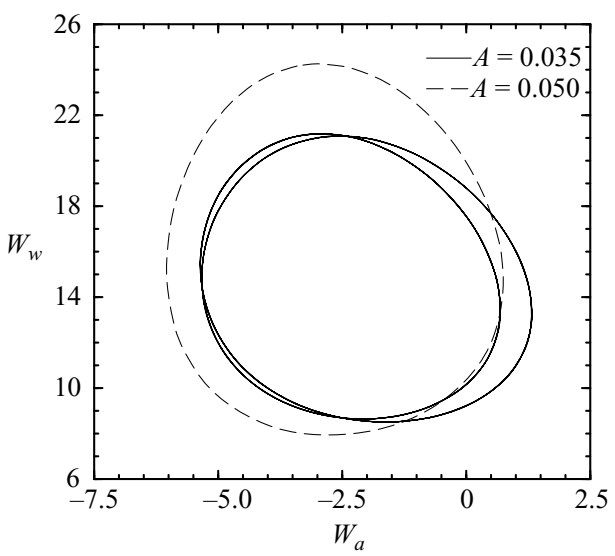

FIGURE 12. Phase portraits in the neighbourhood of the $2: 1$ resonance at $\omega_{f} / \omega_{0}=2.0$ showing a reverse period-doubling bifurcation of limit cycles as $A$ is increased.

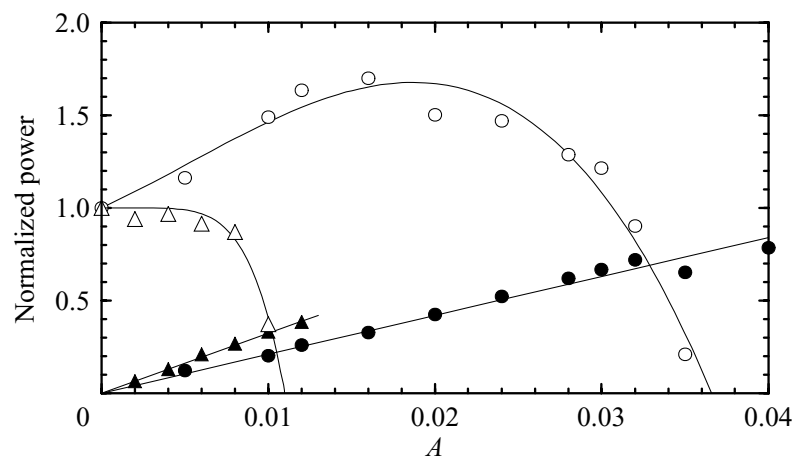

FIGURE 13. Variation of the experimentally measured power (normalized by the power of $L C_{N}$ ) with $A$ in the neighbourhood of the 2:1 resonance horn: the open symbols correspond to the power at the natural frequency $\omega_{0}$ and the filled symbols correspond to the power at the forcing frequency $\omega_{f}$; the circles correspond to $L C_{L}$ inside the horn at $\omega_{f} / \omega_{0} \approx 2$ and the triangles correspond to $Q P$ just outside the horn at $\omega_{f} / \omega_{0} \approx 1.96$.

bifurcation, and following the bifurcation the stroboscopic map of $L C_{L}$ reduces to two period-2 points in the neighbourhood of the slow phases of the invariant circle.

The phase portraits when crossing the period-doubling bifurcation curve separating $L C_{F}$ and $L C_{L}$ are given in figure 12. At $A=0.035$ the phase portrait shows a doublelooped limit cycle $L C_{L}$ with period $4 \pi / \omega_{f}$ inside the horn, and by $A=0.050$ the period-doubling bifurcation has been crossed, and the phase portrait is a single-loop limit cycle $L C_{F}$ synchronous with the forcing.

Figure 13 shows the variation of the power at the natural and forced frequencies for a pair of states in the neighbourhood of the 2:1 resonance. Outside the horn, the power at $\omega_{n}$ (the open triangles) drops off monotonically with increasing $A$ with a rapid decay to zero as the Neimark-Sacker bifurcation is approached, while the power at $\omega_{f}$ (filled triangles) increases linearly with $A$. This behaviour is typical for most $\omega_{f}$ outside resonance horns. Inside the horns, the power at $\omega_{n}$ grows substantially beyond that of the natural limit cycle before gradually decaying to zero as $A$ increases towards the period-doubling bifurcation, as illustrated for the $2: 1$ horn by the open 
circles in the figure. The power at $\omega_{f}$ grows linearly with $A$ as it does outside the horn, as illustrated by the filled circles.

We have analysed in detail the dynamics of the system around the 2:1-resonance horn, finding a very good agreement with analogous periodically forced systems. This shows that both the numerics and the experiments are reliable for this problem. For a forcing amplitude above a critical value (which is small and typically $A \gtrsim 0.04$ ), the oscillatory vortex breakdown flow $L C_{N}$ can be driven to another oscillatory flow $L C_{F}$ at a desired frequency $\omega_{f}$. This result is not particularly surprising; however what is interesting is the spatial distribution of the oscillatory behaviour of $L C_{F}$.

\subsection{Harmonic forcing of $L C_{N}$ : spatial characteristics}

In this section the Reynolds number is fixed at $R e=2800$, and the spatial structure of the oscillations in the flow is investigated as a function of the amplitude and frequency of the forcing. Hot-film measurements are made in the boundary layer at the fixed endwall, so they do not provide any spatial information of the flow. Likewise amplitude equations, such as those used by Gambaudo (1985), Chiffaudel \& Fauve (1987) and Kuznetsov (2004), do not provide any spatial information either.

For small forcing frequency $\left(\omega_{f}=0.1\right)$ figure 5 illustrates that the amplitude of the oscillations near the axis $\left(W_{a}\right)$ and near the wall $\left(W_{w}\right)$ are of the same order of magnitude for the unforced flow $L C_{N}\left(A=0\right.$ ), and the forced flow $L C_{F}$ (at $A=0.03$ ) has similar behaviour. However, for large forcing frequency $\left(\omega_{f}=0.5\right)$, the forced limit cycle resulting from the collapse of the two-torus $Q P$ at the Neimark-Sacker bifurcation has essentially no oscillations near the axis, as illustrated in the sequence of computed phase portraits shown in figure 14. We now employ flow visualization to explore this behaviour experimentally. Figure 15 shows snapshots in the axial region over one forcing period of $L C_{F}$ at a low frequency $\omega_{f}=0.2$ and amplitude $A=0.04$. Comparing with figure 3 , which shows corresponding snapshots of $L C_{N}$, they have qualitatively similar oscillations, as was observed in the computed phase portraits at the lower frequency $\omega_{f}=0.1$ in figure 5 . The limit cycle nature of the flow visualization in figure 15 is confirmed by the hot-film data in figure 16 showing the collapse from $Q P$ to $L C_{F}$ as $A$ is increased at $\omega_{f}=0.2$.

In contrast, for high forcing frequency $\omega_{f}=0.5$, the flow visualization of $L C_{F}$ (figure 17) exhibits a quenching of the oscillations associated with the vortex breakdown bubble. Movies of the $L C_{F}$ dye streak flow visualizations at $\omega_{f}=0.2$ and 0.5 (movies 2 and 3 respectively) are available with the online version of the paper. Even though the flow visualizations of $L C_{F}$ at $\omega_{f}=0.5$ show a stationary recirculation bubble, the hot-film data in figure 18 show that it is in fact a limit cycle synchronous with the forcing. So where is it oscillating? The dye visualization is inadequate to answer this question, because when the dye enters the boundary layer on the rotating disk, it is quickly dispersed and only the flow structure near the axis is clearly observed. To address this, we have also performed flow visualization using fluorescent dye illuminated with a thin laser sheet through a meridional plane, which does allow some visualization of the flow structure in the sidewall boundary layer. Figure 19 shows snapshots of such flow visualizations (the images are cropped to highlight the sidewall boundary layer on the left and the rotating bottom endwall boundary layer, with the essentially steady recirculation zone on the axis providing a reference frame). The snapshots indicate a certain degree of unsteadiness in the bottom left corner region and the sidewall region, and this is more clearly evident in movie 4 (available with the online version of the paper), from which these snapshot were extracted. These flow visualizations provide some guidance as to where $L C_{F}$ at 

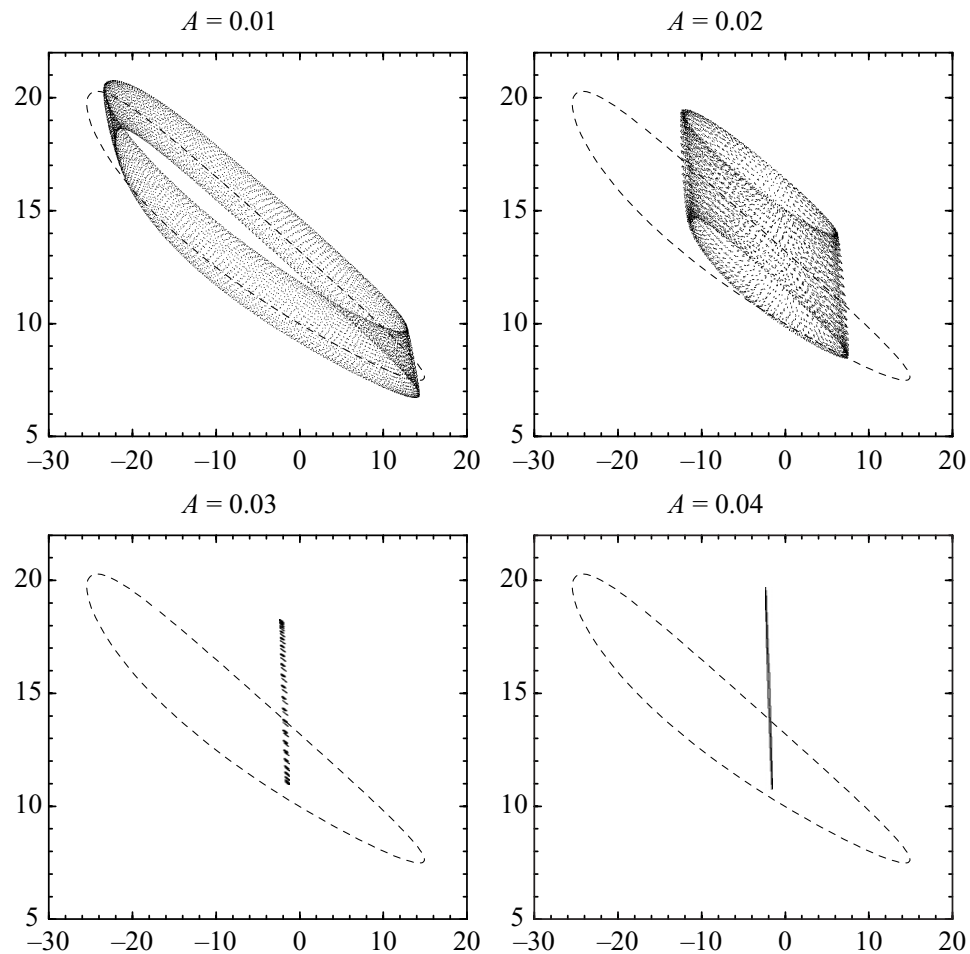

FIGURE 14. Phase portraits (with $W_{a}$ and $W_{w}$ as the horizontal and vertical axes) at $R e=2800$, $H / R=2.5, \omega_{f}=0.5\left(\omega_{f} / \omega_{0} \approx 2.88\right)$ and $A$ as indicated. The dashed circle in the four panels is $L C_{N}$, included for reference.

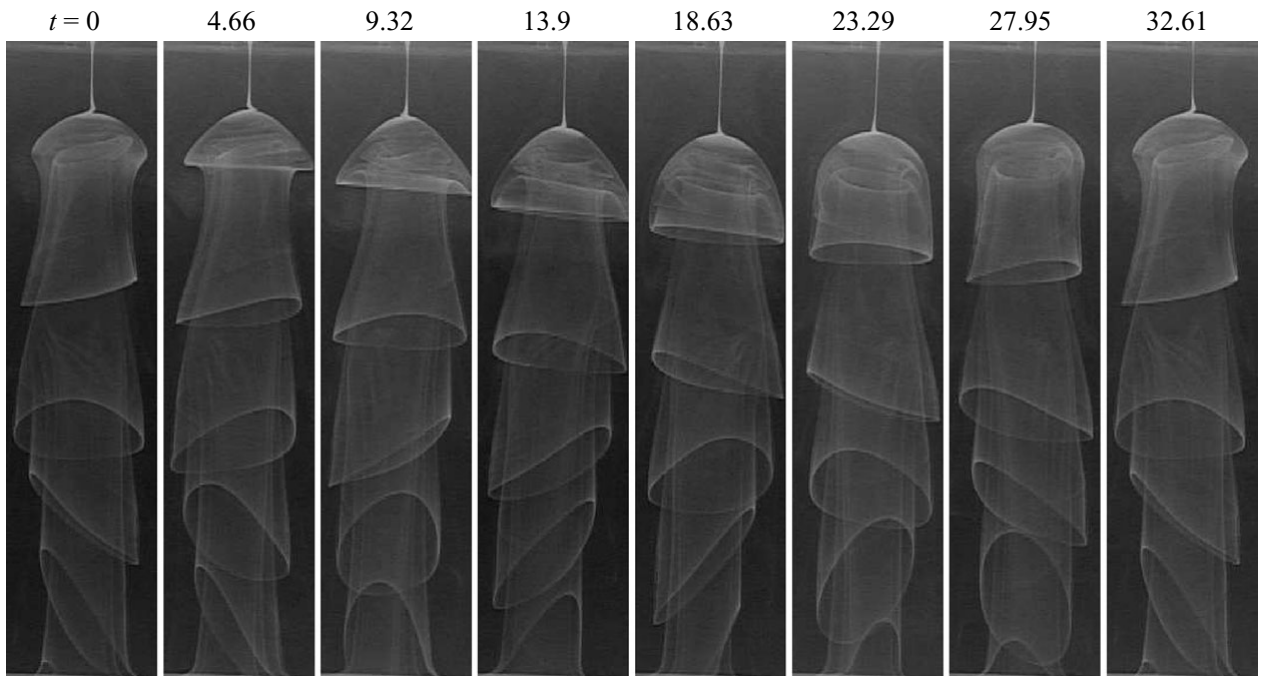

Figure 15. Dye flow visualization of the central core region of a forced state at $H / R=2.5$, $R e=2800, \omega_{f}=0.2$ and $A=0.04$ at roughly equispaced times over one forcing period $T_{f}=2 \pi / \omega_{f}=31.42$ (the time for the first frame has been arbitrarily set to zero). 
(a) $A=0$

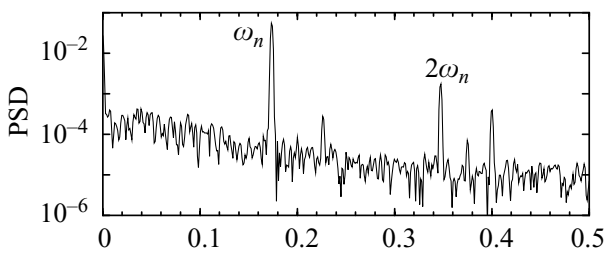

(c) $A=0.010$

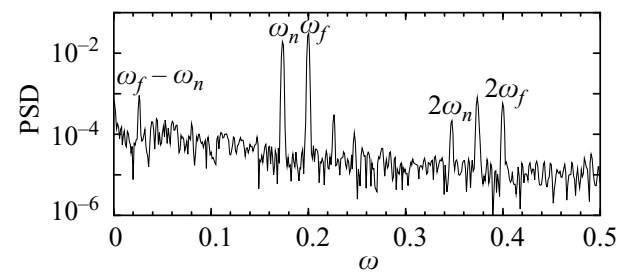

(b) $A=0.005$

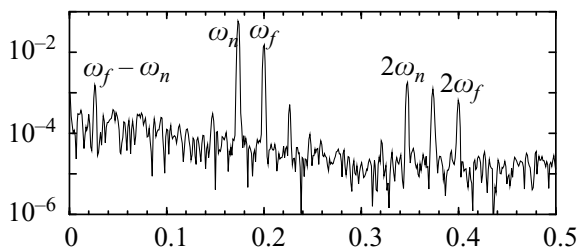

(d) $A=0.015$

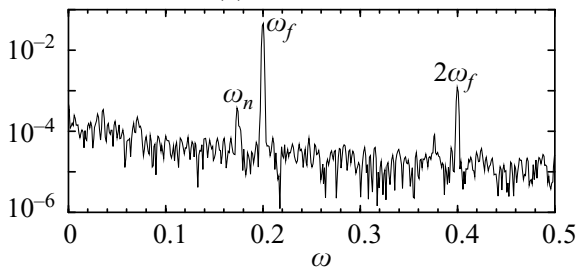

FIGURE 16. Hot-film output time series and corresponding power spectral density for $H / R=2.5, R e=2800$ with forcing frequency $\omega_{f}=0.2$ and forcing amplitude $A$ as indicated.

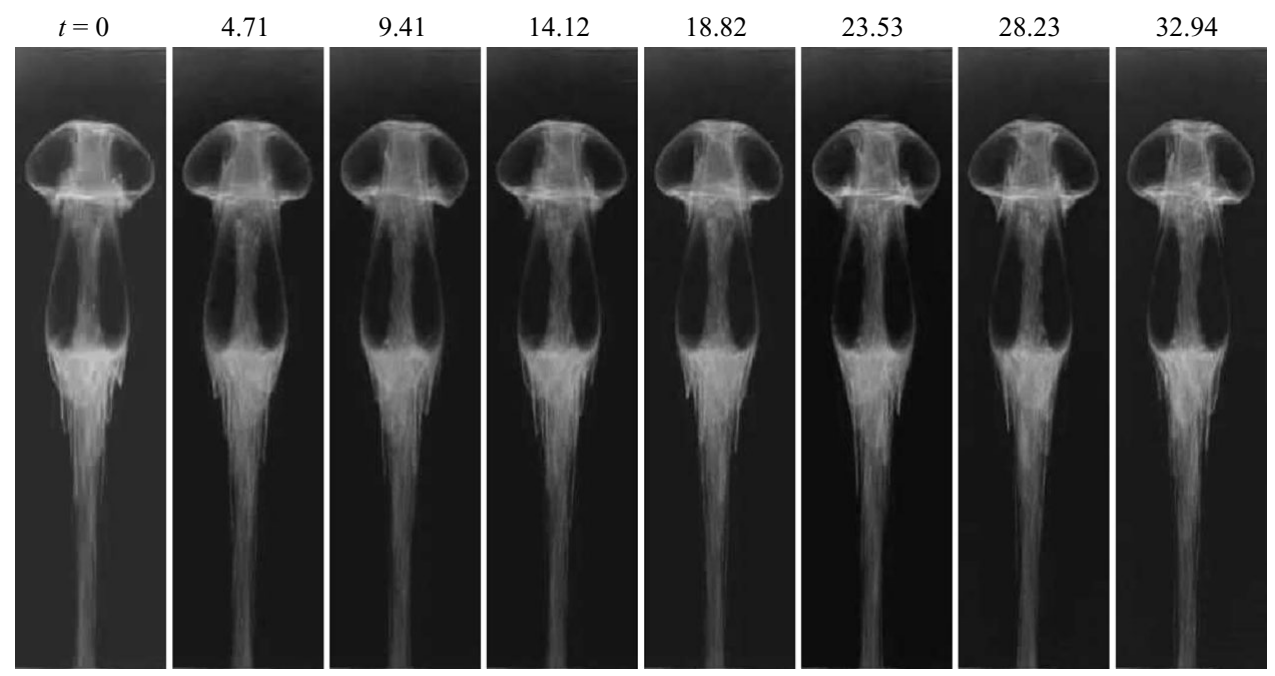

FIGURE 17. Dye flow visualization of the central core region of a forced state at $H / R=2.5$, $R e=2800, \omega_{f}=0.5$ and $A=0.04$ at various times; the forcing period $T_{f}=2 \pi / \omega_{f}=12.57$ (the time for the first frame has been arbitrarily set to zero).

the higher $\omega_{f}$ is oscillating, but the numerical simulations are much better suited to study the spatio-temporal structure of the flow.

Figures 20 and 21 show computed streamlines and contours of the azimuthal vorticity, respectively, of $L C_{F}$ over one forcing period for $\omega_{f}=0.1,0.2,0.3,0.4,0.5$, and 0.6 ; the $\omega_{f}=0.2$ and 0.5 cases correspond to the experimental flow visualizations in figures 15 and 17. The relationship between the unsteady dye streaks in the experiment and the unsteady computed streamlines is discussed in detail in Lopez \& Perry (1992a) for $L C_{N}$, and the same is true for $L C_{F}$ in the present problem. For the $\omega_{f}=0.5$ case, the dye streaks (figure 17) and the computed streamlines in the neighbourhood of 
(a) $A=0.010$

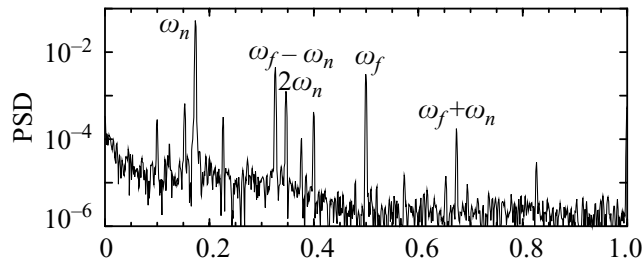

(c) $A=0.020$

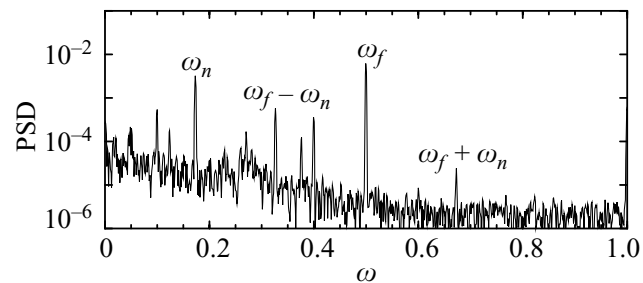

(b) $A=0.015$

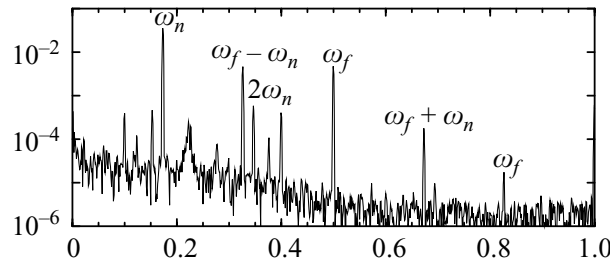

(d) $A=0.040$

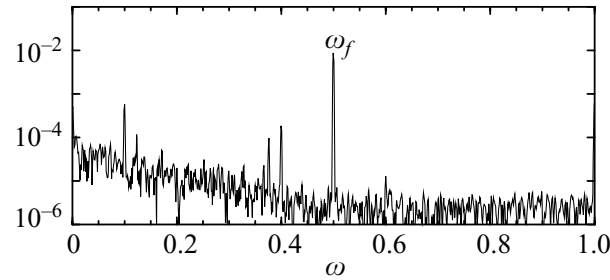

FIGURE 18. Hot-film output time series and corresponding power spectral density for $H / R=2.5, R e=2800$ with forcing frequency $\omega_{f}=0.5$ and forcing amplitude $A$ as indicated.

$t=0$

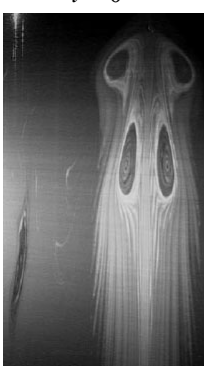

4.77

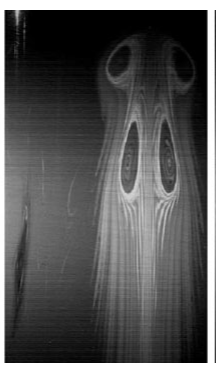

9.54

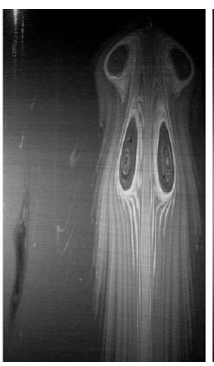

14.31

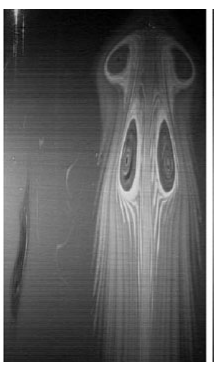

19.08

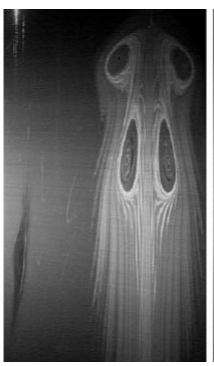

23.85

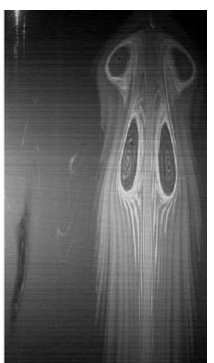

FIGURE 19. Fluorescent dye illuminated with a laser sheet through a meridional plane of $L C_{F}$ at $R e=2800, H / R=2.5, A=0.04$, and $\omega_{f}=0.5$ at various times over about two forcing periods.

the vortex breakdown bubble (figure 20e) coincide quite well, as they should for steady axisymmetric flow. But of course this $L C_{F}$ is not steady. The corresponding hot-film data (figure 18d) establish that this flow is a limit cycle synchronous with the forcing frequency. Neither the streaklines nor the streamlines clearly show where the oscillations are. By plotting contours of the azimuthal component of the vorticity (figure 21e) we clearly see that the oscillations are restricted to the sidewall boundary layer, as was suggested by the laser-sheet visualizations of figure 19 and movie 4 . Movies of the numerically computed streamlines and azimuthal vorticity contours of $L C_{F}$ at $\omega_{f}=0.2$ and 0.5 (movies 5 and 6 respectively) are available with the online version of the paper, illustrating the spatial characteristics of the oscillations just described.

Figures 20 and 21 show the changes in the vortex breakdown bubble and the sidewall boundary layer when $\omega_{f}$ increases from 0.1 to 0.6 in steps of 0.1 . We can clearly appreciate that the transition from an oscillating to a quiescent axial bubble is gradual and continuous with variation in $\omega_{f}$, i.e. there is no bifurcation between 


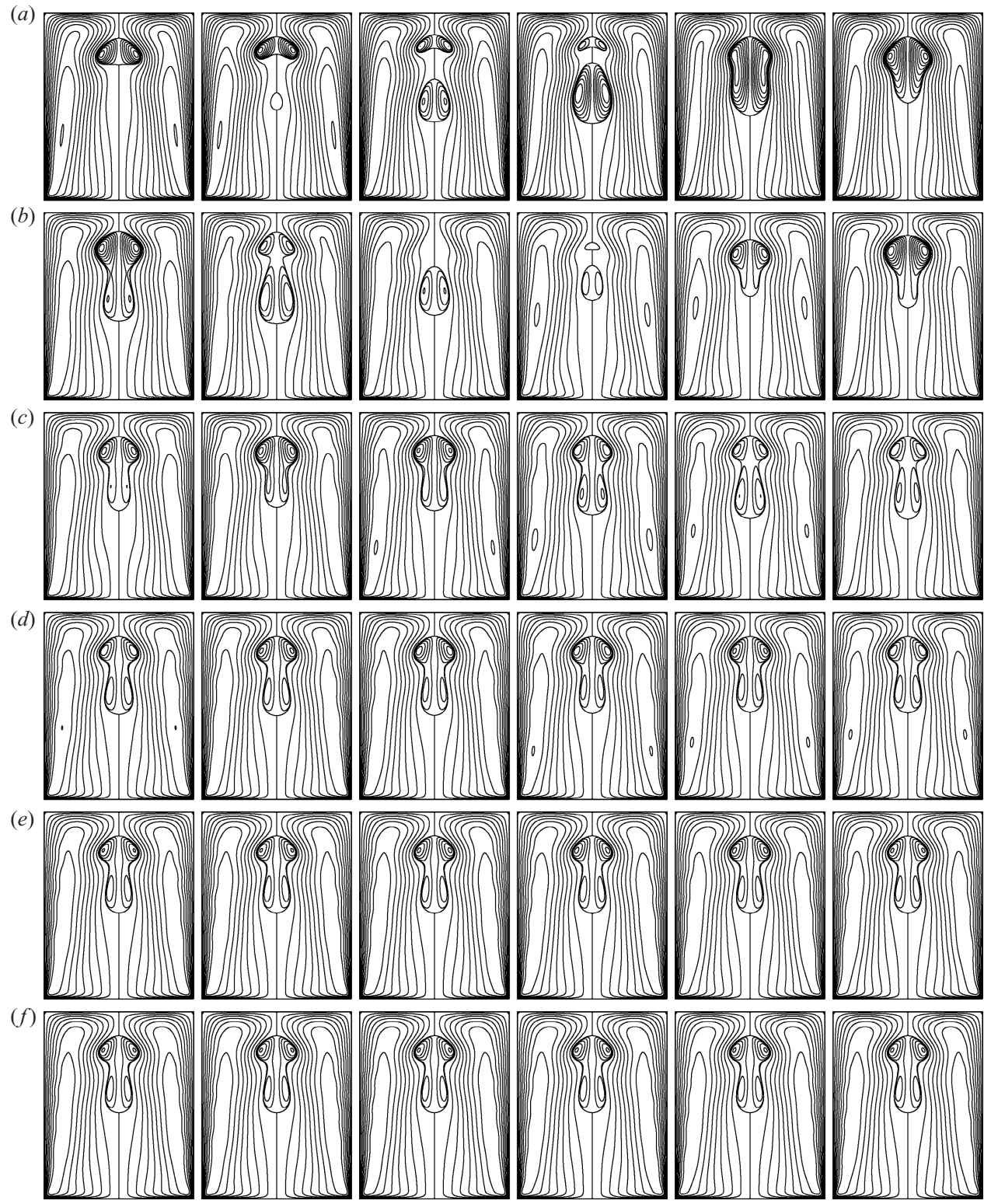

FIGURE 20. Computed streamlines of $L C_{F}$ over one forcing period $2 \pi / \omega_{f}$ (time increases from left to right) at $R e=2800, H / R=2.5, A=0.04$, for increasing values of $\omega_{f}:(a)$ $\omega_{f}=0.1\left(\omega_{f} / \omega_{0} \approx 0.576\right),(b) \omega_{f}=0.2\left(\omega_{f} / \omega_{0} \approx 1.15\right),(c) \omega_{f}=0.3\left(\omega_{f} / \omega_{0} \approx 1.73\right),(d) \omega_{f}=0.4$ $\left(\omega_{f} / \omega_{0} \approx 2.31\right),(e) \omega_{f}=0.5\left(\omega_{f} / \omega_{0} \approx 2.88\right),(f) \omega_{f}=0.6\left(\omega_{f} / \omega_{0} \approx 3.46\right)$.

the oscillatory bubble and the quiescent bubble, all of these solutions are $L C_{F}$ states. Figure 21 shows that while the vortex breakdown oscillations are quenched with increasing $\omega_{f}$, the oscillations in the sidewall boundary layer increase in amplitude and decrease in wavelength. Movie 6 with the online version of the paper shows that these boundary layer oscillations propagate up the sidewall from the rotating disk to the fixed lid (a close examination of the upper sidewall boundary layer region in movie 4 shows similar upward propagating waves). 
(a)

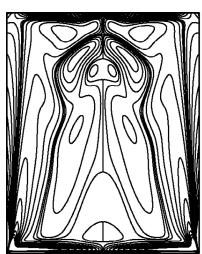

(b)

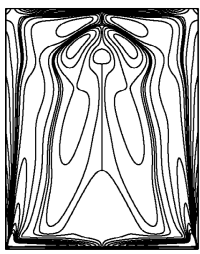

(c)

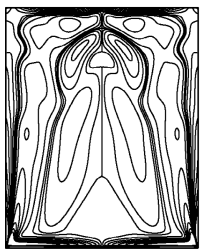

(d)

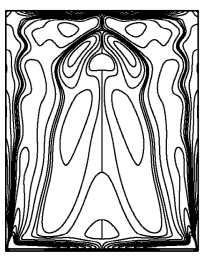

(e)

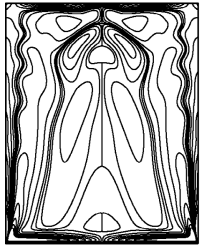

(f)

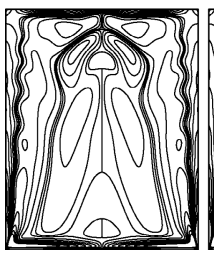

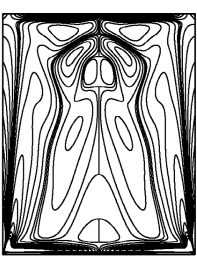
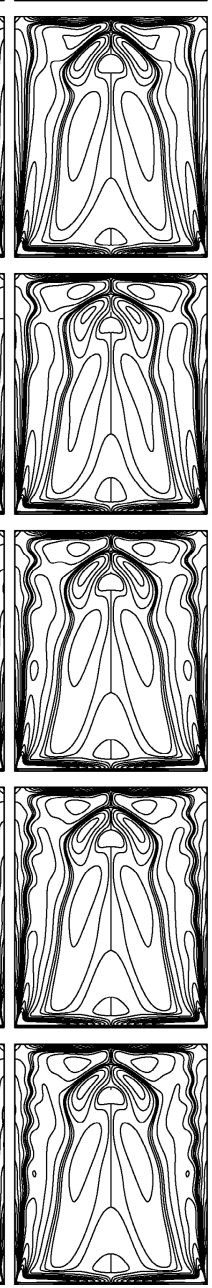
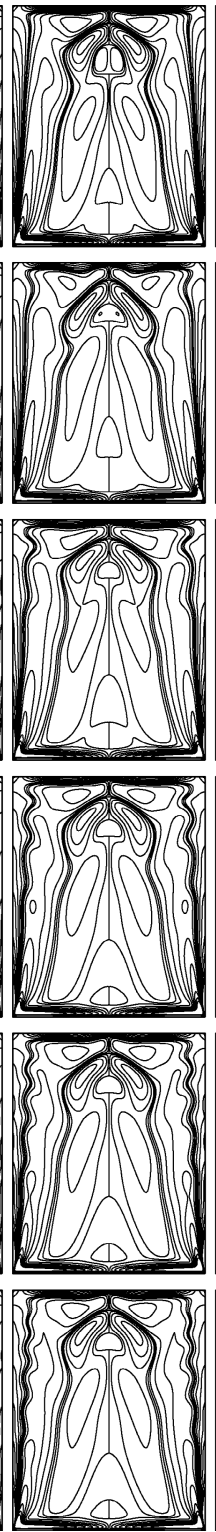
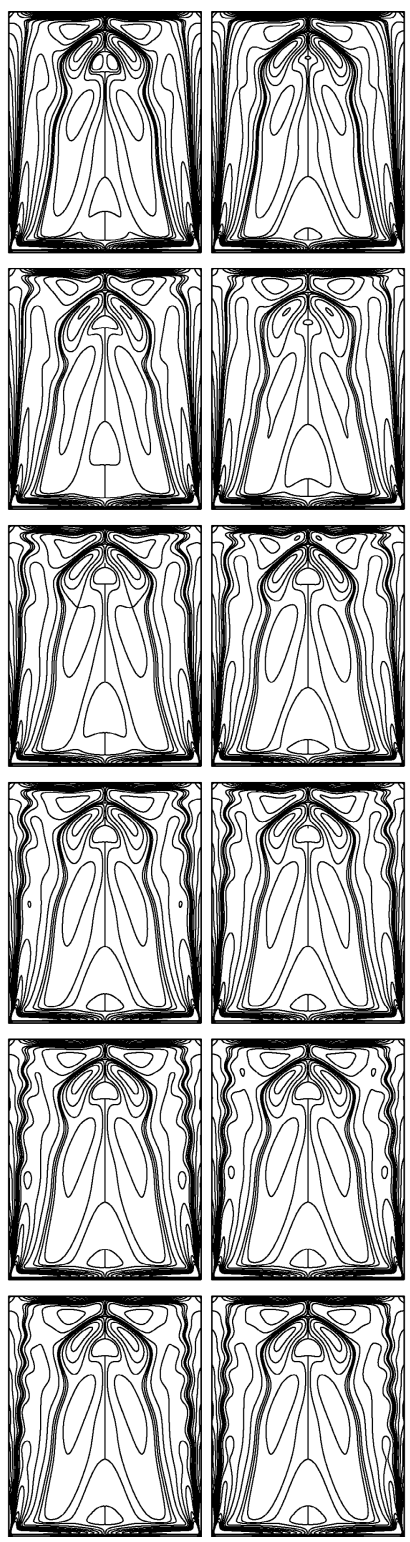
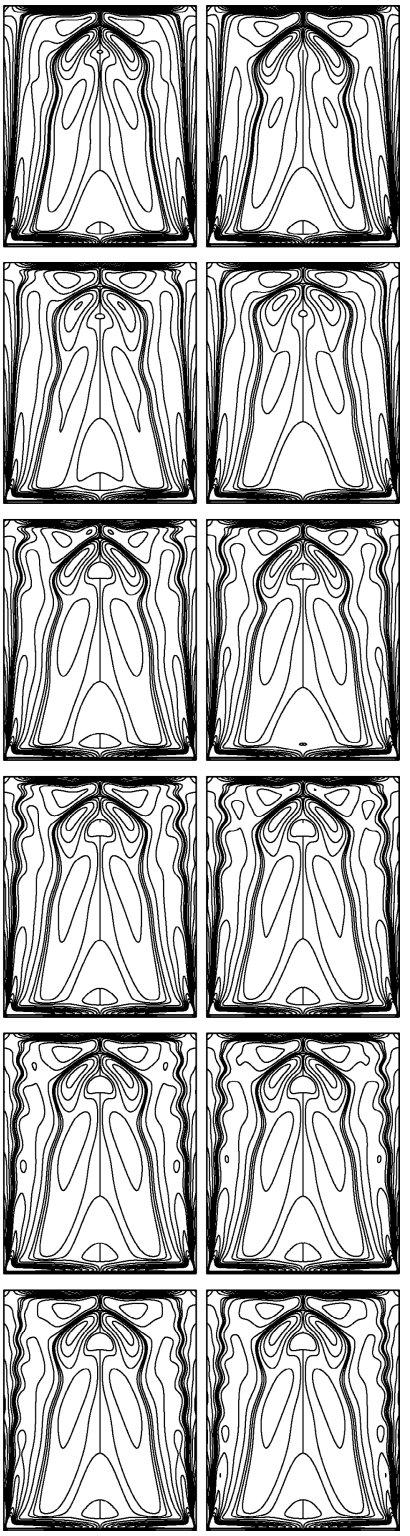

FIGURE 21. Computed azimuthal vorticity contours of $L C_{F}$ over one forcing period $2 \pi / \omega_{f}$ at $R e=2800, H / R=2.5, A=0.04$, for increasing values of $\omega_{f}:(a) \omega_{f}=0.1\left(\omega_{f} / \omega_{0} \approx 0.576\right),(b)$ $\omega_{f}=0.2\left(\omega_{f} / \omega_{0} \approx 1.15\right),(c) \omega_{f}=0.3\left(\omega_{f} / \omega_{0} \approx 1.73\right),(d) \omega_{f}=0.4\left(\omega_{f} / \omega_{0} \approx 2.31\right),(e) \omega_{f}=0.5$ $\left(\omega_{f} / \omega_{0} \approx 2.88\right),(f) \omega_{f}=0.6\left(\omega_{f} / \omega_{0} \approx 3.46\right)$.

\section{Discussion and conclusions}

We have conducted an experimental and numerical analysis of the harmonically modulated unsteady vortex breakdown flow in a cylindrical container of aspect ratio $H / R=2.5$, in the region where the flow remains axisymmetric (from $R e \approx 2710$ up to $R e \approx 3000$ ). We have explored a wide range of frequency forcing values, for moderate forcing amplitudes; figure 7 shows the explored region and the bifurcations we have observed. The quasi-periodic flow $Q P$ having both the natural frequency $\omega_{n}$ of the 

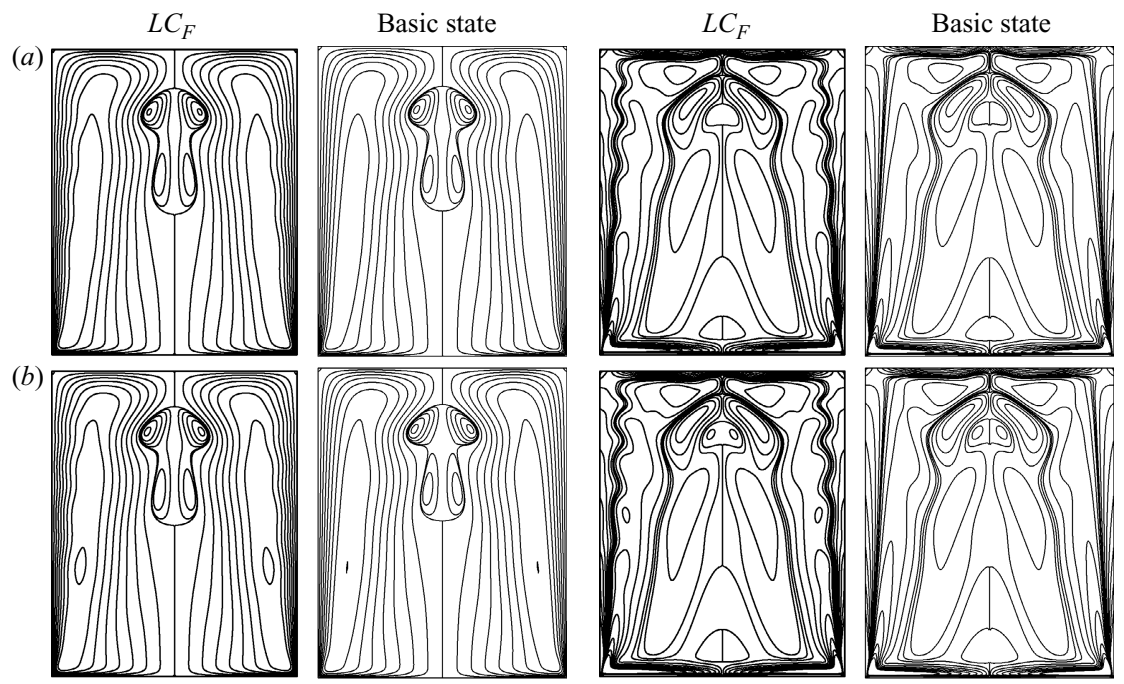

(c)
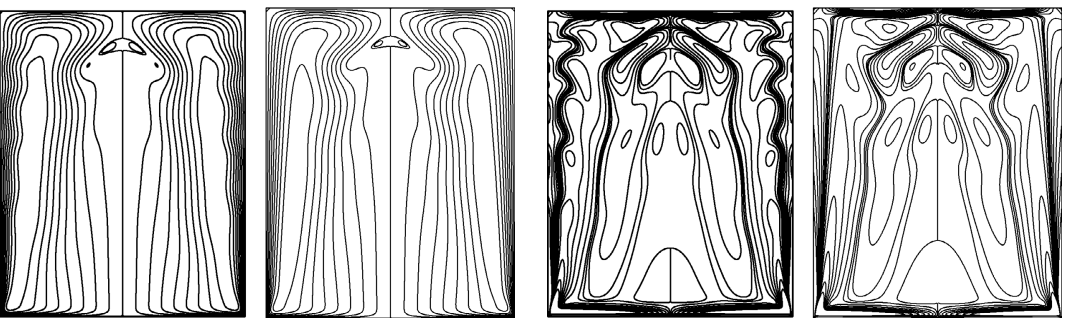

FiguRE 22. Streamlines (right two panels) and contours of the azimuthal vorticity (left two panels) for $L C_{F}$ at $A=0.04$ and $\omega_{f}=0.5$ (showing a snapshot in time) and for the (unstable) basic state which was computed using arclength continuation and finite difference in Lopez et al. (2001). (a) $R e=2800$, (b) $R e=3000$, (c) $R e=4000$.

unsteady vortex breakdown bubble and the forcing frequency $\omega_{f}$, exists between two Neimark-Sacker bifurcations curves (the axis $A=0$ and the solid line in figure 7); a variety of resonance horns emerge from both curves, connecting them. As there are no symmetries in this problem, except the rotational $S O(2)$ symmetry which is not broken for the parameter values studied, the dynamic behaviour is generic, and so we have also found period-doubling regions. The dynamics observed are very rich, and we have explored some of them in detail, in particular the 2:1 resonance, where theoretical results covering the whole region between the two Neimark-Sacker curves are available in the literature, and we have found a very good agreement with these.

What is particularly novel in the present study is the spatial characteristics of the forced limit cycle $L C_{F}$ that exists for forcing amplitudes above the second NeimarkSacker curve. For forcing frequencies less than about twice the natural frequency, the oscillations of the vortex breakdown bubble are enhanced, whereas for forcing frequencies greater than about twice the natural frequency, the oscillations of the breakdown bubble are completely quenched and all the oscillations in $L C_{F}$ are restricted to the sidewall boundary layer region. Furthermore, the quenched $L C_{F}$ structure is essentially the same as the structure of the steady axisymmetric base state (except of course near the sidewall); see figure 22(a) which compares the snapshots of $L C_{F}$ at $R e=2800, A=0.04$ and $\omega_{f}=0.5$ with the (unstable) basic state at $R e=2800$ which was computed using arclength continuation and finite difference in Lopez et al. 
(2001). Note that at $R e=2800$ the base state is only unstable to a single Hopf mode, $L C_{N}$. By $R e=3000$, the basic state has undergone a second Hopf bifurcation to $L C_{S}$. Forcing the system in the axisymmetric subspace at $R e=3000$, also with $A=0.04$ and $\omega_{f}=0.5$, results in an $L C_{F}$ which, apart from the sidewall boundary layer region, also coincides remarkably well with the unstable base state (see figure $22 b$ ). At $R e=4000$ the base state has undergone a third Hopf bifurcation, and for the same forcing amplitude and frequency $\left(A=0.04\right.$ and $\left.\omega_{f}=0.5\right)$ all three Hopf modes are quenched and the resulting $L C_{F}$ still coincides remarkably well with the unstable base state (see figure $22 c$ ).

This open-loop control study has shown that the low-amplitude modulations can either enhance the oscillations of the vortex breakdown bubble (for low frequencies) or quench them (for high frequencies). Enhancing the oscillations can be beneficial in some applications where mixing is desired, such as swirl combustion chambers. The results indicate that high-frequency modulations of unsteady vortex breakdown drive the system to the unstable basic state in the vortex core region. This suggests that the basic state, which exists for all Reynolds numbers, can be used as a goal in a closed-loop control strategy. This would be interesting to explore in other applications where unsteady vortex breakdown is prevalent, such as the tail buffeting problem.

This work was supported by the National Science Foundation grant DMS-0505489, the Spanish Ministry of Education and Science grant FIS2004-01336, and Catalonian Government grant SGR-00024. Y. D. C. gratefully acknowledges the support from the Directorate of Research and Development, Defense Science and Technology Agency, Singapore, under the Flow Control Program POD-0103935. Computational resources of ASU's Fulton HPCI are greatly appreciated.

\section{REFERENCES}

Arnold, V. I. 1983 Geometrical Methods in the Theory of Ordinary Differential Equations. Springer. Arrowsmith, D. K. \& Place, C. M. 1990 An Introduction to Dynamical Systems. Cambridge University Press.

Blackburn, H. M. 2002 Three-dimensional instability and state selection in an oscillatory axisymmetric swirling flow. Phys. Fluids 14, 3983-3996.

Blackburn, H. M. \& Lopez, J. M. 2000 Symmetry breaking of the flow in a cylinder driven by a rotating end wall. Phys. Fluids 12, 2698-2701.

Blackburn, H. M. \& Lopez, J. M. 2002 Modulated rotating waves in an enclosed swirling flow. J. Fluid Mech. 465, 33-58.

Brons, M., Shen, W. Z., Sorensen, J. N. \& Zhu, W. 2007 The influence of imperfections on the flow structure of steady vortex breakdown bubbles. J. Fluid Mech. 578, 453-466.

Chiffaudel, A. \& Fauve, S. 1987 Stong resonance in forced oscillatory convection. Phys. Rev. A 35, 4004-4007.

Crawford, J. D. \& KNobloch, E. 1991 Symmetry and symmetry-breaking bifurcations in fluid dynamics. Annu. Rev. Fluid Mech. 23, 341-387.

Delery, J. M. 1994 Aspects of vortex breakdown. Prog. Aerospace Sci. 30, 1-59.

EsCUDIER, M. P. 1984 Observations of the flow produced in a cylindrical container by a rotating endwall. Exps. Fluids 2, 189-196.

Escudier, M. P. 1988 Vortex breakdown: Observations and explanations. Prog. Aerospace Sci. 25, 189-229.

Fornberg, B. 1998 A Practical Guide to Pseudospectral Methods. Cambridge University Press.

Gallaire, F., Chomaz, J.-M. \& Huerre, P. 2004 Closed-loop control of vortex breakdown: a model study. J. Fluid Mech. 511, 67-93.

Gambaudo, J. M. 1985 Perturbation of a Hopf bifurcation by an external time-periodic forcing. J. Diffl Equat. 57, 172-199. 
Gelfgat, A. Y., Bar-Yoseph, P. Z. \& Solan, A. 2001 Three-dimensional instability of axisymmetric flow in a rotating lid-cylinder enclosure. J. Fluid Mech. 438, 363-377.

Hall, P. 1972 Vortex breakdown. Annu. Rev. Fluid Mech. 4, 195-218.

Herrada, M. A. \& Shtern, V. 2003 Control of vortex breakdown by temperature gradients. Phys. Fluids 15, 3468-3477.

Hourigan, K., Graham, L. W. \& Thompson, M. C. 1995 Spiral streaklines in pre-vortex breakdown regions of axisymmetric swirling flows. Phys. Fluids 7, 3126-3128.

Hughes, S. \& Randriamampianina, A. 1998 An improved projection scheme applied to pseudospectral methods for the incompressible Navier-Stokes equations. Intl J.Num. Meth. Fluids 28, 501-521.

Husain, H. S., Shtern, V. \& Hussain, F. 2003 Control of vortex breakdown by addition of near-axis swirl. Phys. Fluids 15, 271-279.

Iooss, G. \& Adelmeyer, M. 1998 Topics in Bifurcation Theory and Applications, 2nd edn. World Scientific.

Khalil, S., Hourigan, K. \& Thompson, M. C. 2006 Response of unconfined vortex breakdown to axial pulsing. Phys. Fluids 18, 038102.

KNOBLOCH, E. 1996 Symmetry and instability in rotating hydrodynamic and magnetohydrodynamic flows. Phys. Fluids 8, 1446-1454.

KuZnetsov, Y. A. 2004 Elements of Applied Bifurcation Theory, 3rd edn. Springer.

Leibovich, S. 1978 The structure of vortex breakdown. Annu Rev. Fluid Mech. 10, 221-246.

Lopez, J. M. 1990 Axisymmetric vortex breakdown. Part 1. Confined swirling flow. J. Fluid Mech. 221, 533-552.

Lopez, J. M. 2006 Rotating and modulated rotating waves in transitions of an enclosed swirling flow. J. Fluid Mech. 553, 323-346.

Lopez, J. M., CuI, Y. D. \& LiM, T. T. 2006 An experimental and numerical investigation of the competition between axisymmetric time-periodic modes in an enclosed swirling flow. Phys. Fluids 18, 104106.

Lopez, J. M., Marques, F. \& Sanchez, J. 2001 Oscillatory modes in an enclosed swirling flow. J. Fluid Mech. 439, 109-129.

Lopez, J. M. \& Perry, A. D. 1992 a Axisymmetric vortex breakdown. Part 3. Onset of periodic flow and chaotic advection. J. Fluid Mech. 234, 449-471.

Lopez, J. M. \& Perry, A. D. $1992 b$ Periodic axisymmetric vortex breakdown in a cylinder with a rotating end wall. Phys. Fluids A 4, 1871.

Lucca-Negro, O. \& O’Doherty, T. 2001 Vortex brekdown: a review. Prog. Energy Combust. Sci. 27, 431-481.

Lugt, H. J. \& Aвboud, M. 1987 Axisymmetric vortex breakdown with and without temperature effects in a container with a rotating lid. J. Fluid Mech. 179, 179-200.

Mercader, I., Net, M. \& Falqués, A. 1991 Spectral methods for high order equations. Comput. Meth. Appl. Mech. Engng 91, 1245-1251.

Mitchell, A. M. \& Délery, J. 2001 Research into vortex breakdown control. Prog. Aerospace Sci. 37, 385-418.

Mununga, L., Hourigan, K., Thompson, M. C. \& Leweke, T. 2004 Confined flow vortex breakdown control using a small rotating disk. Phys. Fluids 16, 4750-4753.

Orszag, S. A. \& Patera, A. T. 1983 Secondary instability of wall-bounded shear flows. J. Fluid Mech. 128, 347-385.

Schilder, F. \& Peckham, B. B. 2007 Computing Arnol'd tongue scenarios. J. Comput. Phys. 220, 932-951.

Sotiropoulos, F. \& Ventikos, Y. 2001 The three-dimensional structure of confined swirling flows with vortex breakdown. J. Fluid Mech. 426, 155-175.

Sotiropoulos, F., Webster, D. R. \& LACKey, T. C. 2002 Experiments on Lagrangian transport in steady vortex-breakdown bubbles in a confined swirling flow. J. Fluid Mech. 466, 215-248.

Stevens, J. L., Lopez, J. M. \& CANTwell, B. J. 1999 Oscillatory flow states in an enclosed cylinder with a rotating endwall. J. Fluid Mech 389, 101-118.

Thompson, M. C. \& Hourigan, K. 2003 The sensitivity of steady vortex breakdown bubbles in confined cylinder flows to rotating lid misalignment. J. Fluid Mech. 496, 129-138.

VENTIKos, Y. 2002 The effect of imperfections on the emergence of three-dimensionality in stationary vortex breakdown bubbles. Phys. Fluids 14, 13-16. 